\title{
MEASURES ON BANACH MANIFOLDS AND SUPERSYMMETRIC QUANTUM FIELD THEORY
}

\author{
JONATHAN WEITSMAN
}

\begin{abstract}
We show how to construct measures on Banach manifolds associated to supersymmetric quantum field theories. These measures are mathematically well-defined objects inspired by the formal path integrals appearing in the physics literature on quantum field theory. We give three concrete examples of our construction. The first example is a family $\mu_{P}^{s, t}$ of measures on a space of functions on the two-torus, parametrized by a polynomial $P$ (the Wess-Zumino-Landau-Ginzburg model). The second is a family $\mu_{\mathcal{G}}^{s, t}$ of measures on a space $\mathcal{G}$ of maps from $\mathbb{P}^{1}$ to a Lie group (the Wess-Zumino-Novikov-Witten model). Finally we study a family $\mu_{M, G}^{s, t}$ of measures on the product of a space of connections on the trivial principal bundle with structure group $G$ on a three-dimensional manifold $M$ with a space of $\mathfrak{g}$-valued three-forms on $M$.

We show that these measures are positive, and that the measures $\mu_{\mathcal{G}}^{s, t}$ are Borel probability measures. As an application we show that formulas arising from expectations in the measures $\mu_{\mathcal{G}}^{s, 1}$ reproduce formulas discovered by Frenkel and Zhu in the theory of vertex operator algebras. We conjecture that a similar computation for the measures $\mu_{M, S U(2)}^{s, t}$, where $M$ is a homology threesphere, will yield the Casson invariant of $M$.
\end{abstract}

\section{INTRODUCTION}

During the past two decades techniques and ideas arising from quantum field theory have played an increasing role in geometry and topology. The work of the school of mathematical physicists led by E. Witten on applications of supersymmetric quantum field theory and later of string theory to problems in mathematics has in many cases led to results that can be stated as mathematically precise conjectures, and often rigorously proved as theorems. However, the methods used by the physicists, among which formal path-integral methods of integration over infinite-dimensional spaces are prominent, have not in the main been accessible to mathematical interpretation; for actual computation one proceeds by inspired analogy to finite-dimensional cases and by physical intuition. The purpose of this paper is to produce measures on infinite-dimensional spaces associated to supersymmetric quantum field theories which maintain the formal analogies to finite dimensions used by the physicists, but which are mathematically well-defined.

1.1. Finite dimensional analogs of supersymmetric quantum field theory. In this paper we give a construction of measures on Banach manifolds associated to supersymmetric quantum field theories. In order to approach this problem, we begin with finite-dimensional analogs of these theories and the associated measures. In the simplest case, given a map of finite-dimensional vector spaces, we give three constructions that coincide: That of the degree of the map, of the pull-back by this map of a Gaussian differential form of top degree, and of a measure obtained by pushforwards by local inverses. The third construction is the one which we generalize to Banach manifolds. The physics literature makes use of formal analogy to finite dimensions to work with the first and second constructions only.

Supported in part by NSF grant DMS 04/05670.

September 16, 2018. 
1.1.1. Measures arising from maps of vector spaces. Let $V, W$ be real, $n$-dimensional Hermitian vector spaces. Let $\eta_{W}$ denote the invariant volume form on $W$, and let $\xi \in H^{n}(W)$ denote the differential form $\xi=\frac{1}{(2 \pi)^{n / 2}} \exp \left(-|w|^{2} / 2\right) \eta_{W}$. Given a proper smooth map $f: V \longrightarrow W$, the pullback $f^{*} \xi$ gives a signed measure on $V$. In terms of the natural volume form $\eta_{V}$ this measure is given by

$$
f^{*} \xi=\frac{1}{(2 \pi)^{n / 2}} \exp \left(-|f|^{2} / 2\right) \operatorname{det}(\nabla f) \eta_{V} .
$$

If $f$ grows rapidly at infinity the integral of $f^{*} \xi$ converges and gives the Leray-Schauder degree of the map $f$, so that if 0 is a regular value of $f$, we have

$$
\int_{V} f^{*} \xi=\sum_{x \in f^{-1}(0)} \epsilon_{x}
$$

where $\epsilon_{x}=\operatorname{det}\left(\left.\nabla f\right|_{x}\right) /\left|\operatorname{det}\left(\left.\nabla f\right|_{x}\right)\right|$. Equation (1.2) can be seen to follow formally from the expression (1.1) by replacing $f$ by $t f$ and then letting $t \longrightarrow \infty$. The determinant appearing in (1.1) can be viewed as arising from a Berezin integration, so that the integral is a finite-dimensional analog of supersymmetric quantum field theory. The fact that supersymmetric field theories are analogs of integrals of the type (1.1) and are related to the degree of the map corresponding to $f$ is due to Witten in [4, 44].

Formulas similar to (1.1) and (1.2) hold where $V, W$ are complex Hermitian vector spaces and $f: V \longrightarrow W$ is a proper smooth map. If $W$ is a complex vector space of complex dimension $n$, the Euclidean volume form on $W$ can be written as $\eta_{W}=\omega^{n} / n$ !, where $\omega$ is a two-form on $W$ given in terms of a set $\left(w_{1}, \ldots, w_{n}\right)$ of holomorphic coordinates on $W$ by $\omega=\sum_{i} d w_{i} \wedge d \bar{w}_{i}$. It follows that if $f: V \longrightarrow W$ is holomorphic and $x \in V$ is a regular point of $f$ then $\operatorname{det}\left(\left.\nabla f\right|_{x}\right) /\left|\operatorname{det}\left(\left.\nabla f\right|_{x}\right)\right|=1$.

An expression for the measure given by $f^{*} \xi$ can also be given as follows. Let $x \in V$ be a regular point of $f$, and let $U$ be a neighborhood of $x$ where $f$ is invertible; denote this local inverse by $g$. Let $\mu_{W}$ be the Gaussian measure on $W$ corresponding to $\xi$, and consider its restriction to $f(U)$. We define a measure on $U$ by

$$
\mu_{U}:=g_{*}\left(\left.\mu_{W}\right|_{f(U)}\right) .
$$

This gives a measure $f^{*} \mu_{W}$ on $V$ by taking the union over all neighborhoods $U$ and by assigning measure zero to the singular points of $f$. The measure $f^{*} \mu_{W}$ can be obtained from the signed measure associated to $f^{*} \xi$ by taking absolute values of the determinant of $\nabla f$; in other words, for any $\phi \in C_{c}(V)$,

$$
\frac{1}{(2 \pi)^{n / 2}} \int \phi \exp \left(-|f|^{2} / 2\right) \operatorname{det}(\nabla f) \eta_{V}=f^{*} \mu_{W}(\phi \text { Phase } \operatorname{det}(\nabla f))
$$

where Phase $\operatorname{det}(\nabla f)(x)$ is given by Phase $\operatorname{det}(\nabla f)(x):=\operatorname{det}\left(\left.\nabla f\right|_{x}\right) /\left|\operatorname{det}\left(\nabla f_{x}\right)\right|$ if $x$ is a regular point of $f$ and is extended arbitrarily to the singular set of $f$ where the measure $f^{*} \mu_{W}$ is zero.

Two features of the finite-dimensional case will recur in the generalization of this construction to Banach manifolds. These are the subtlety involved in the definition of the phase of the determinant of $\nabla f$, and the issue of the finiteness of the measure $f^{*} \mu_{W}$. In the case of Banach manifolds, the measure analogous to $f^{*} \mu_{W}$ will have a straightforward construction, but the phase of the determinant may not be well-defined. This phenomenon is known in the physics literature as the appearance of an anomaly; the geometry underlying anomalies was studied by Atiyah-Singer [5] and Quillen 30. Similarly, in finite dimensions, the finiteness of the measure $f^{*} \mu_{W}$ depends on the growth properties of $f$ at infinity, and must be studied by hard analysis. The same will be true in the case of Banach manifolds. 
1.1.2. Measures arising from sections of vector bundles. Finite-dimensional analogs of supersymmetric quantum field theories also arise in a slightly different context. Let $M^{n}$ be a compact Riemannian manifold, let $E \longrightarrow M$ be an $n$-dimensional Hermitian vector bundle over $M$, and let $\nabla$ be a connection on $E$. Let $s: M \longrightarrow E$ be a section of $E$. In this case Mathai and Quillen [26] give an expression for the Euler number $\chi(E)$ of $E$ as

$$
\chi(E)=\frac{1}{(2 \pi)^{n / 2}} \int_{M} \exp \left(-|s|^{2} / 2\right) \operatorname{det}(\nabla s) \sum_{k=1}^{n} C_{k} ;
$$

here $C_{k}$ is a sequence of terms involving $\nabla s$ and and the curvature of $E$. In terms of local coordinates the Mathai-Quillen formula is given by the integral of a sum of such terms multiplied by a phase of a determinant with respect to a measure of the type defined in the previous section.

1.2. Outline of the paper. The physics literature on topological quantum field theory is concerned with formal infinite-dimensional integrals corresponding morally to the differential form (1.1), where the map $f: V \longrightarrow W$ is replaced by a map $\mathcal{F}$ of infinite dimensional spaces. It then seeks to apply ideas from differential geometry (such as localization), as well as novel techniques, to gain insight about the solutions of the equation $\mathcal{F}=0$, and more generally about the formal properties of the underlying measure. While some of the results of this approach can be stated mathematically and sometimes verified by various mathematical methods, there have been so far no satisfactory constructions of measures on infinite dimensional spaces which would approach the physicists' formal constructions.

In this paper we will describe a method of constructing measures on Banach manifolds analogous to the pullback measures $f^{*} \mu_{W}$ in a number of examples motivated by supersymmetric quantum field theories. Starting with a supersymmetric model based on a map $\mathcal{F}$ of infinite-dimensional spaces, we will find a family of Banach manifolds $X_{s}, s>s_{0}$, a Banach space $Y$, and a family of maps $\mathcal{F}_{s}: X_{s} \longrightarrow Y$, where the set $\mathcal{F}_{s}=0$ is independent of $s$ and matches formally the set $\mathcal{F}_{0}=\mathcal{F}=0$. The Banach space $Y$ in our examples will be equipped with a white noise measurethe infinite-dimensional analog of the Gaussian measure $\mu_{W}$ above. We will construct an analog of the pullback measure $f^{*} \mu_{W}$ and of the phase of the determinant. We do not know of a general theorem that would give convergence of the integral of the phase of the determinant; instead we study some examples paralleling supersymmetric quantum field theories in the literature. In these examples we can give specific conjectures about the finiteness of the measure and the value of the integral of the phase of the determinant. In one example we will be able to prove the analogs of these conjectures.

This paper is structured as follows. In Section 2, we first recall some facts about white noise measures. These are the infinite dimensional analogs of the Gaussian measures $\mu_{W}$ above. We then study maps $\mathcal{F}: X \longrightarrow Y$ where $X$ is a Banach manifold and $Y$ is a Banach space equipped with a white noise measure. If $\mathcal{F}: X \longrightarrow Y$ is a differentiable (that is, $C^{1}$ ) map, we obtain a measure on $X$ analogous to the measure $f^{*} \mu_{W}$ arising in (1.3). This is done in Proposition 2.4.

In Section 3, we give examples of this construction of measures where the maps are motivated by supersymmetric quantum field theories appearing in the physics literature. In these examples we also give a construction of the phase of the determinant using a generalization of Fredholm determinants. We study three families of measures. The first family is denoted $\mu_{P}^{s, t}$, where $P$ is a polynomial in one variable and $s, t$ are parameters. These are measures on a Banach space of functions on the torus, and are related to objects known as the Wess-Zumino-Landau-Ginzburg models (Theorem 11). We next consider a family $\mu_{\mathcal{G}}^{s, t}$ of measures on a group $\mathcal{G}$ of maps from $\mathbb{P}^{1}$ to a complex Lie group; these measures are related to the Wess-Zumino-Novikov-Witten models (Theorem 21). Finally, given a compact Lie group $G$ and a three-manifold $M$, we consider measures $\mu_{M, G}^{s, t}$ on the product of a space of connections on a principal $G$ bundle on $M$ with a space of $\mathfrak{g}$ valued three-forms on $M$. These measures are related to three-dimensional quantum gauge theory 
(Theorem 3). We conjecture that the measures $\mu_{P}^{s, t}$ and $\mu_{M, G}^{s, t}$ extend to Borel probability measures. We also give precise conjectures about the values of the integrals of the phases of appropriate determinants (Conjecture 3.11 and Conjecture 3.19). In the case of three-dimensional gauge theory the conjecture is that $\mu_{M, S U(2)}^{s, t}$ should yield the Casson invariant of homology three-spheres.

At the end of this section we briefly describe how our methods can be used in a few other examples of maps of Banach manifolds inspired by supersymmetric quantum field theories.

In Section 4 we turn to the measures $\mu_{\mathcal{G}}^{s, t}$, where we are able to prove analogs of the conjectures we have made about $\mu_{P}^{s, t}$ and $\mu_{M, G}^{s, t}$. Using results of Atiyah and Bott [3], we show that $\mu_{\mathcal{G}}^{s, t}$ is a Borel probability measure on the space $\mathcal{G}$ (Theorem 4 ). We then compute (Theorem 5 ) some expectations of functions in this measure to recover formulas discovered by Frenkel and Zhu [12] in the context of vertex operator algebras.

\section{Maps And measures on Banach manifolds}

In this section we consider some general constructions of measures on Banach manifolds. We begin with Gaussian measures and then present a method of producing non-Gaussian measures from Gaussian measures and maps of Banach manifolds. ${ }^{1}$

2.1. White Noise and Gaussian Measures. Let $M^{d}$ be a smooth compact Riemannian manifold and let $V \longrightarrow M$ be a smooth Hermitian vector bundle over $M$. Let $\Gamma(V)$ denote the space of smooth sections of $V$. The Hermitian metric on $V$ and the Riemannian structure on $M$ give rise to an $L_{2}$ inner product $<,>$ on $\Gamma(V)$. For $\lambda \in \mathbb{R}$, let $H_{\lambda}(M)$ denote the distributions on $M$ of Sobolev class $\lambda$ and let $\Gamma_{\lambda}(V)=\Gamma(V) \otimes H_{\lambda}(M)$ denote the space of sections of $V$ of Sobolev class $\lambda$. Let $\alpha$ be a connection on $V$, and let $\Delta_{\alpha}$ be the corresponding Laplacian on sections of $V$. This Laplacian gives rise to a hermitian inner product on $\Gamma(V)$ given by $\left\langle s, s^{\prime}\right\rangle_{\lambda}=\left\langle s,\left(-\Delta_{\alpha}+1\right)^{\lambda} s^{\prime}>\right.$ for $s, s^{\prime} \in \Gamma(V)$. This inner product extends to $\Gamma_{\lambda}(V)$ and makes $\Gamma_{\lambda}(V)$ into a separable Hilbert space. If $\lambda>0$ the space $\Gamma_{-\lambda}(V)$ may be identified with the dual of $\Gamma_{\lambda}(V)$; we continue to denote the pairing of $\Gamma_{-\lambda}(V)$ with $\Gamma_{\lambda}(V)$ by $<$, > . If $\lambda=0$ we write $\Gamma_{0}(V)=L_{2}(M, V)$, and the pairing $<$, $>$ gives rise to the $L_{2}$ metric on $\Gamma_{0}(V)$.

If $\lambda<-d / 2$, the space $\Gamma_{\lambda}(V)$ comes equipped with a finite Borel measure $\mu_{V}$ called white noise measure, which is characterized by the following property:

Let $\phi \in \Gamma_{-\lambda}(V)$ and let $E_{\phi}: \Gamma_{\lambda}(V) \longrightarrow \mathbb{C}$ denote the function given by

$$
E_{\phi}(\sigma)=\exp (i \operatorname{Re}<\phi, \sigma>)
$$

Then

$$
\mu_{V}\left(E_{\phi}\right)=\exp \left(-\frac{1}{2}\|\phi\|_{L_{2}}^{2}\right)
$$

In particular, $\mu_{V}(1)=1$

Given $t>0$, let $R_{t}: \Gamma_{\lambda}(V) \longrightarrow \Gamma_{\lambda}(V)$ be the scaling function $R_{t}(v)=t v$. We write $\mu_{V}^{t}=$ $\left(R_{t^{-1}}\right)_{*} \mu_{V}$ for the scaled white noise measure. Then for every $\epsilon>0$, we have $\mu_{V}^{t}\left(B_{\epsilon}(0)\right)>0$ for $t$ sufficiently large.

We now define more general Gaussian measures. Choose $s \in \mathbb{R}$. Then $\left(-\Delta_{\alpha}+1\right)^{-s}: \Gamma_{\lambda}(V) \longrightarrow$ $\Gamma_{\lambda+2 s}(V)$ is an isomorphism. Let $\lambda<-d / 2$ as before; then we may push the white noise measure $\mu_{V}^{t}$ forward to obtain a Gaussian measure $\mu_{V, s}^{t}:=\left(\left(-\Delta_{\alpha}+1\right)^{-s}\right)_{*} \mu_{V}^{t}$ on $\Gamma_{\nu}(V)$ where $\nu<2 s-d / 2 .^{2}$

\footnotetext{
${ }^{1}$ In this paper we consider both the case of complex Banach spaces and that of real Banach spaces. The results presented in this section hold in either the real or complex category, and all formulas should be interpreted in the appropriate setting.

${ }^{2}$ In the literature these measures are usually regarded as living on the spaces $Z_{\lambda}(V)$ of sections of $V$ of $Z y g m u n d$ class $\lambda<2 s-d / 2$. However, an extension of the Sobolev embedding theorem (see [20], Proposition 8.6.10) shows that there exist continuous inclusions

$$
Z_{\lambda+\epsilon}(V) \subset \Gamma_{\lambda}(V) \subset Z_{\lambda-d / 2}(V)
$$
}


Proposition 2.2 (Cameron-Martin). Let $\lambda<2 s-d / 2$ and let $v \in \Gamma(V)$. Let $T_{v}: \Gamma_{\lambda}(V) \longrightarrow \Gamma_{\lambda}(V)$ be the translation given by $T_{v}(a)=v+a$. Then $\left(T_{v}\right)_{*} \mu_{V, s}^{t}$ is absolutely continuous with respect to $\mu_{V, s}^{t}$, and the Radon Nikodym derivative may be written as

$$
\frac{d\left(T_{v}\right)_{*} \mu_{V, s}^{t}}{d \mu_{V, s}^{t}}=\exp \left(t^{2}<\left(-\Delta_{\alpha}+1\right)^{2 s} v, \cdot>-\frac{1}{2} t^{2}\|v\|_{2 s}^{2}\right) .
$$

The translate of the measure $\mu_{V, s}^{t}$ by an arbitrary element $v \in \Gamma_{\lambda}(V)$ may not be absolutely continuous with respect to $\mu_{V, s}^{t}$.

2.2. Pullbacks of measures by differentiable maps and the main construction. Let $X$ be a separable metric space. Let $\left\{\left(U_{\alpha}, \mu_{\alpha}\right)\right\}_{\alpha \in \mathcal{A}}$ be a collection of pairs $\left(U_{\alpha}, \mu_{a}\right)$ where $\left\{U_{\alpha}\right\}_{\alpha \in \mathcal{A}}$ is an open cover of $X$ and $\mu_{\alpha}$ is a finite Borel measure on $U_{\alpha}$ for each $\alpha$. Suppose that for each $\alpha, \beta \in \mathcal{A}$,

$$
\left.\mu_{\alpha}\right|_{U_{\alpha} \cap U_{\beta}}=\left.\mu_{\beta}\right|_{U_{\alpha} \cap U_{\beta}} .
$$

Since $X$ is Lindelöf, we obtain a $\sigma$-finite measure $\mu$ on $X .{ }^{3}$ We will call such a measure a Borel probability measure if $\infty>\mu(X)>0$.

Let $X, Y$ be separable Banach manifolds and let $\mu$ be a finite Borel measure on $Y$. Suppose $\mathcal{F}: X \longrightarrow Y$ is a differentiable map, and let $U \subset X$ be the (open) set of points $p \in X$ such that $\delta \mathcal{F}_{p}$ is an isomorphism. For each $p \in U$, we can find a neighborhood $U_{p}$ of $p$ where $\left.\mathcal{F}\right|_{U_{p}}$ is invertible. Denote this local inverse by $\mathcal{G}_{p}$, and define a finite Borel measure $\nu_{U_{p}}$ on $U_{p}$ by

$$
\nu_{U_{p}}:=\mathcal{G}_{p_{*}}\left(\left.\mu\right|_{\mathcal{F}\left(U_{p}\right)}\right) .
$$

Then the collection $\left\{\left(U_{p}, \nu_{U_{p}}\right)\right\}$ gives a measure $\nu$ on $U$. Let $i: U \longrightarrow X$ be the inclusion. We define the measure $\mathcal{F}^{*} \mu$ on $X$ by $\mathcal{F}^{*} \mu=i_{*} \nu$.

We therefore obtain the following result, which is our main tool for constructing measures on Banach manifolds.

Recall that a differentiable map $\mathcal{F}: X \longrightarrow Y$ of separable Banach spaces is a Fredholm map if $\left.\delta \mathcal{F}\right|_{x}$ is Fredholm for all $x \in X$.

Proposition 2.4. Let $X, Y$ be separable Banach manifolds. Let $\mu$ be a finite Borel measure on $Y$. Let $\mathcal{F}: X \longrightarrow Y$ be a differentiable map. Then the map $\mathcal{F}$ induces a $\sigma$-finite Borel measure $\mathcal{F}^{*} \mu$ on $X$, supported on the open set $U \subset X$ where $\delta \mathcal{F}$ is an isomorphism.

In particular, let $M^{d}$ be a compact Riemannian manifold of dimension $d$, and let $V$ be a vector bundle over $M$. Let $\lambda<-d / 2$, and let $\mathcal{F}: X \longrightarrow \Gamma_{\lambda}(V)$ be a differentiable map. Suppose that $\mathcal{F}$ is a Fredholm map and that ind $\left.\delta \mathcal{F}\right|_{x}=0$ for all $x \in X$. Then for any $t>0$ the map $\mathcal{F}$ induces a $\sigma$-finite Borel measure $\mathcal{F}^{*} \mu_{V}^{t}$ on $X$, supported on the set of regular points of $\mathcal{F}$.

Suppose that there exists $p \in X$ which is a regular point of $\mathcal{F}$ and satisfies $\mathcal{F}(p)=0$. Then for $t$ sufficiently large, there exists a neighborhood $W \subset X$ of $p$ such that

$$
\infty>\mathcal{F}^{*} \mu_{V}^{t}(W)>0 .
$$

The methods we have used in this section cannot decide whether $\mathcal{F}^{*} \mu_{V}^{t}$ gives a Borel probability measure on $X$. This has to be checked by analysis in each example. We will do this in one example in Section 4.

for any $\epsilon>0$, so that our measures can just as well be regarded as living on $\Gamma_{\lambda}(V)$. This will be convenient for various purposes in this paper.

${ }^{3}$ To do this, extend the measure to any countable subcover of the cover $\left\{U_{\alpha}\right\}_{\alpha \in \mathcal{A}}$. This extension is independent of the choice of countable subcover. 


\section{Examples of measures on Banach manifolds}

In this section we give examples of maps which can be used to construct measures on Banach manifolds by the construction of Proposition 2.4. These maps are inspired by formal path integrals appearing in the physics literature.

3.1. Some analytical preliminaries. In this section we collect some analytical facts we will use in our constructions.

3.1.1. Sobolev bounds. In order to study nonlinear maps on Banach manifolds, we will use the following result on the regularity of a product of distributions.

Proposition 3.1. [see e.g. [20], Prop. 8.3.1] ${ }^{4}$

Let $f_{1} \in H_{s_{1}}\left(M^{d}\right), f_{2} \in H_{s_{2}}\left(M^{d}\right)$. Suppose $s_{1}+s_{2} \geq 0$. Then $f_{1} f_{2} \in H_{s}\left(M^{d}\right)$ if

$$
s \leq s_{i}, i=1,2 \text { and } s<s_{1}+s_{2}-d / 2 .
$$

The product $f_{1} f_{2}$ is bounded by

$$
\left\|f_{1} f_{2}\right\|_{s}<K\left\|f_{1}\right\|_{s_{1}}\left\|f_{2}\right\|_{s_{2}} .
$$

where $K$ is a constant depending on $s, s_{1}, s_{2}$.

This result immediately implies a similar result for sections of vector bundles of the appropriate Sobolev classes.

3.1.2. Schatten classes and regularized determinants. To define phases of determinants of some of the operators we will encounter, we will use regularized determinants [1], 37.

Let $\mathcal{H}$ be a separable Hilbert space, and let $\mathcal{L}(\mathcal{H})$ denote the ring of bounded operators on $\mathcal{H}$. For $k \geq 1$, write $\mathcal{S}_{k}(\mathcal{H})$ for the $k$-th Schatten ideal of $\mathcal{H}$; that is, the set of compact operators $K: \mathcal{H} \longrightarrow \mathcal{H}$ such that $\left(K^{*} K\right)^{k / 2}$ is trace class. The space $\mathcal{S}_{k}(\mathcal{H})$ is a two-sided ideal of $\mathcal{L}(\mathcal{H})$. The function $K \longrightarrow\|K\|_{k}:=\operatorname{tr}\left(K^{*} K\right)^{k / 2}$ defines a norm on $\mathcal{S}_{k}(\mathcal{H})$ which makes $\mathcal{S}_{k}(\mathcal{H})$ into a Banach space, and the inclusion $\mathcal{S}_{k}(\mathcal{H}) \longrightarrow \mathcal{L}(\mathcal{H})$ is continuous. Similarly, if $k<k^{\prime}, \mathcal{S}_{k}(\mathcal{H}) \subset \mathcal{S}_{k^{\prime}}(\mathcal{H})$, and the inclusion $\mathcal{S}_{k}(\mathcal{H}) \longrightarrow \mathcal{S}_{k^{\prime}}(\mathcal{H})$ is continuous.

An example of an element of a Schatten ideal is given as follows. Let $M$ be a compact Riemannian manifold of dimension $d$ and let $V$ be a Hermitian vector bundle over $M$. Let $\alpha$ be a connection on $V$, let $\left(-\Delta_{\alpha}+1\right): \Gamma_{\lambda+2}(V) \longrightarrow \Gamma_{\lambda}(V)$ be the Laplacian, and let $j: \Gamma_{\lambda+2}(V) \longrightarrow \Gamma_{\lambda}(V)$ denote the inclusion. Then

$$
j \circ\left(-\Delta_{\alpha}+1\right)^{-1} \in \mathcal{S}_{r}\left(\Gamma_{\lambda}(V)\right)
$$

for all $r>d / 2$.

Recall that if $K \in \mathcal{S}_{1}(\mathcal{H})$, the Fredholm determinant $\operatorname{det}(1+K)$ is defined [17] by the convergent series

$$
\operatorname{det}(1+K):=\sum_{n=0}^{\infty} \operatorname{tr} \wedge^{n} K .
$$

This construction was generalized to operators in $\mathcal{S}_{k}(\mathcal{H})$ by Poincaré [29]; we follow Simon [37. Given $K \in \mathcal{L}(\mathcal{H})$, define $R_{k}(K) \in \mathcal{L}(\mathcal{H})$ by

$$
R_{k}(K):=\left[(1+K) \exp \left(\sum_{n=1}^{k-1} \frac{(-K)^{n}}{n}\right)\right]-1 .
$$

\footnotetext{
${ }^{4}$ We will use the bound (3.2) only in the situation where $s_{1}, s_{2}>d / 2$; in other words, when the Sobolev spaces $H_{s_{i}}\left(M^{d}\right)$ are spaces of continuous functions.
} 
Simon shows that if $K \in \mathcal{S}_{k}(\mathcal{H})$, then $R_{k}(K) \in \mathcal{S}_{1}(\mathcal{H})$. Define the regularized determinant det ${ }_{k}(1+$ $K)$ by $^{5}$

$$
\operatorname{det}_{k}(1+K):=\operatorname{det}\left(1+R_{k}(K)\right) .
$$

The function $\operatorname{det}_{k}(1+\cdot): \mathcal{S}_{k}(\mathcal{H}) \longrightarrow \mathbb{C}$ is continuous. If $K$ is trace class, the regularized determinant is related to the Fredholm determinant $\operatorname{det}(1+K)$ by

$$
\operatorname{det}_{k}(1+K)=\operatorname{det}(1+K) \exp \left(\sum_{n=1}^{k-1} \frac{(-1)^{n}}{n} \operatorname{tr} K^{n}\right) .
$$

The regularized determinant has multiplicativity properties which can be deduced from (3.7) and the multiplicativity of the Fredholm determinant by approximating elements of the Schatten classes by trace-class operators (which are dense in $\mathcal{S}_{k}(\mathcal{H})$ ). From these multiplicativity properties it follows that if $1+K$ is invertible, the regularized determinant $\operatorname{det}_{k}(1+K)$ is nonzero.

3.2. Wess-Zumino-Landau-Ginzburg models on the torus. Let $\Sigma=S^{1} \times S^{1}$ be the torus, equipped with a Kähler structure, and let $V=\Sigma \times \mathbb{C} \longrightarrow \Sigma$ be the trivial bundle over $\Sigma$. If $\lambda \in \mathbb{R}$, the space $\Gamma_{\lambda}(V)$ is given by $\Gamma_{\lambda}(V)=H_{\lambda}(\Sigma)$. Choose $\lambda<-1$ and $s>-\lambda / 2$. Let $\mu_{V}$ be the white noise measure on $\Gamma_{\lambda}(V)$. By the Sobolev embedding theorem, the space $\Gamma_{\lambda+2 s+1}(V)$ consists of continuous functions.

Let $P$ be a polynomial in one variable. Define $\mathcal{F}_{s}: \Gamma_{\lambda+2 s+1}(V) \longrightarrow \Gamma_{\lambda}(V)$ by

$$
\mathcal{F}_{s}(\phi)=(-\Delta+1)^{s}\left(\partial \phi+\overline{P^{\prime}(\phi)}\right) .
$$

Theorem 1. The map $\mathcal{F}_{s}$ is well-defined and differentiable. Its derivative is a Fredholm operator of index 0.

Therefore, for each $t>0$, the pullback measure

$$
\mu_{P}^{s, t}:=\mathcal{F}_{s}^{*} \mu_{V}^{t}
$$

exists and is a $\sigma$-finite measure on $\Gamma_{\lambda+2 s+1}(V)$, supported on the set of regular points of $\mathcal{F}_{s}$. If the polynomial $P^{\prime}$ has a transversal zero at $y \in \mathbb{C}$, then for $t$ sufficiently large, there exists a neighborhood $W$ of the constant function $\phi_{0}=y$ such that

$$
\infty>\mu_{P}^{s, t}(W)>0 \text {. }
$$

Proof. Proposition 3.1 shows that the map $\mathcal{F}_{s}$ is well-defined and differentiable. The derivative $\delta \mathcal{F}_{s}$ is a compact perturbation of the isomorphism $(-\Delta+1)^{s}(\partial+1): \Gamma_{\lambda+2 s+1}(V) \longrightarrow \Gamma_{\lambda}(V)$, and so is Fredholm of index 0. The existence of the measure follows by Proposition 2.4,

If $y \in \mathbb{C}$ is a point where $P^{\prime}(y)=0$ and $P^{\prime \prime}(y) \neq 0$, the constant function $\phi_{0}:=y$ satisfies $\mathcal{F}_{s}\left(\phi_{0}\right)=0$, and $\left.\delta \mathcal{F}_{s}\right|_{\phi_{0}}=(-\Delta+1)^{s}\left(\partial+P^{\prime \prime}(y)\right)$ is an isomorphism. The positivity of the measure $\mathcal{F}_{s}^{*} \mu_{V}^{t}$ in a neighborhood of $\phi_{0}$ for $t$ sufficiently large follows, again, by Proposition 2.4

We make the following conjecture.

Conjecture 3.9. The measure $\mu_{P}^{s, t}$ satisfies

$$
\mu_{P}^{s, t}(1)<\infty
$$

for $t$ sufficiently large.

Suppose the polynomial $P^{\prime}$ has a transversal zero. Then for $t$ sufficiently large, Conjecture 3.9 implies that the measure $\mu_{P}^{s, t}$ is a Borel probability measure on $\Gamma_{\lambda+2 s+1}(V)$.

${ }^{5}$ The motivation for this definition is Plemelj's formula (valid when $K \in \mathcal{S}_{1}(\mathcal{H})$ and $\|K\|_{1}<1$ )

$$
\operatorname{det}(1+K)=\exp (\operatorname{tr} \log (1+K)) \text {. }
$$

The appearance of $R_{k}(K)$ in (3.6) amounts to removing the first $k-1$ terms from the power series for $\log (1+K)$ in Plemelj's formula. The remaining terms are multiples of $K^{n}$ where $n \geq k$ and so are trace class if $K \in S_{k}(\mathcal{H})$. 
3.2.1. The phase of the determinant of $\delta \mathcal{F}_{s}$. The derivative $\left.\delta \mathcal{F}_{s}\right|_{\phi}$ is given by the Fredholm operator

$$
D_{\phi}:=(-\Delta+1)^{s}\left(\partial+\overline{P^{\prime \prime}(\phi)}\right): \Gamma_{\lambda+2 s+1}(V) \longrightarrow \Gamma_{\lambda}(V) .
$$

If $P^{\prime}$ is nonconstant and $y$ is a point where $P^{\prime \prime}(y) \neq 0$, then if $\phi_{0}:=y$ is the constant function, $D_{\phi_{0}}$ is an isomorphism. Then $\left(D_{\phi_{0}}\right)^{-1} D_{\phi}: \Gamma_{\lambda+2 s+1}(V) \longrightarrow \Gamma_{\lambda+2 s+1}(V)$ can be written as a compact perturbation of the identity

$$
\left(D_{\phi_{0}}\right)^{-1} D_{\phi}=1+K\left(\phi_{0}, \phi\right)
$$

where

$$
K\left(\phi_{0}, \phi\right)=\left(\partial+P^{\prime \prime}\left(\phi_{0}\right)\right)^{-1} \circ j \circ M\left(\overline{P^{\prime \prime}(\phi)}-\overline{P^{\prime \prime}\left(\phi_{0}\right)}\right) .
$$

Here for $\psi \in \Gamma_{\lambda+2 s+1}(V)$, we have denoted by

$$
M(\psi): \Gamma_{\lambda+2 s+1}(V) \longrightarrow \Gamma_{\lambda+2 s+1}(V)
$$

the operator given by multiplication by $\psi, j: \Gamma_{\lambda+2 s+1}(V) \longrightarrow \Gamma_{\lambda+2 s}(V)$ is the inclusion, and $\left(\partial+P^{\prime \prime}\left(\phi_{0}\right)\right)^{-1}$ is the inverse of the isomorphism

$$
\partial+P^{\prime \prime}\left(\phi_{0}\right): \Gamma_{\lambda+2 s+1}(V) \longrightarrow \Gamma_{\lambda+2 s}(V) .
$$

Since $\|M(\psi)\| \leq\|\psi\|_{2 s+\lambda+1}$ by Proposition 3.1, the estimate (3.3) shows that

$$
K\left(\phi_{0}, \phi\right) \in \mathcal{S}_{3}\left(\Gamma_{\lambda+2 s+1}(V)\right)
$$

for all $\phi$. The regularized determinant $\operatorname{det}_{3}\left(1+K\left(\phi_{0}, \phi\right)\right)$ is therefore well-defined for all $\phi$, and is nonzero at all regular points of $\mathcal{F}_{s}$. Let $U$ be the set of regular points of $\mathcal{F}_{s}$. Define $\Psi\left(\phi_{0}, \cdot\right): U \longrightarrow S^{1}$ by

$$
\Psi\left(\phi_{0}, \phi\right)=\frac{\operatorname{det}_{3}\left(1+K\left(\phi_{0}, \phi\right)\right)}{\left|\operatorname{det}_{3}\left(1+K\left(\phi_{0}, \phi\right)\right)\right|} .
$$

Morally, the function $\Psi\left(\phi_{0}, \phi\right)$ gives the ratio of the "phase of the determinant of $\left.\delta \mathcal{F}_{s}\right|_{\phi}$ " to the "phase of the determinant of $\left.\delta \mathcal{F}_{s}\right|_{\phi_{0}}$." This method of defining determinants is used in the physics literature; by analogy with the case of maps of finite-dimensional vector spaces given in equations (1.2) and (1.4) in the introduction, we make the following conjecture.

Conjecture 3.11. Assume that Conjecture 3.9 holds. Then for t sufficiently large,

$$
\left|\mu_{P}^{s, t}\left(\Psi\left(\phi_{0}, \cdot\right)\right)\right|=(\operatorname{deg} P-1) .
$$

Morally this conjecture says that for $t$ large, the measure $\mu_{P}^{s, t}$ is concentrated at the solutions of the nonlinear equation $\mathcal{F}_{s}=0$.

Remark 3.12. The formal path integral for the Wess-Zumino-Landau-Ginzburg model appearing in the physics literature corresponds to formula (1.1) applied (formally) to $\mathcal{F}_{0}$, and should morally count the solutions of the partial differential equation $\mathcal{F}_{0}=0$. Since the solutions of the equation $\mathcal{F}_{s}=0$ are exactly those of the equation $\mathcal{F}_{0}=0$, the computations associated formally with the path integral corresponding to $\mathcal{F}_{0}$ should morally be replicated for the measure $\mu_{P}^{s, t}$. The supersymmetric field theory corresponding to these path integrals was constructed using classical methods of constructive quantum field theory in [21, and the resulting partition function does count (for generic $P$ ) the number of solutions of $\mathcal{F}_{0}=0$, which correspond to constant functions with values given by the zeros of $P$.

The idea of using an infinite-dimensional degree to find solutions of nonlinear partial differential equations goes back to Leray-Schauder, who considered proper maps. The case of measures induced by nonlinear maps on $\Gamma_{\lambda}(V)$ where $V$ is a vector bundle over a one-dimensional manifold was considered in [15] using the methods of [23, 32, 24, 14, In this case the pullback measure on $\Gamma_{\lambda}(V)$ is a perturbation of a Gaussian measure, and the analog of our operator $K$ is Hilbert-Schmidt. This is not the case if the underlying manifold is of dimension greater than one. 
The subtlety in the definition of the phase of the determinant arising from the need to use regularized determinants was to my knowledge first observed in the physics literature. ${ }^{6}$

3.3. Wess-Zumino-Novikov-Witten models on $\mathbb{P}^{1}$. Let $G$ be a compact semisimple Lie group. We consider the corresponding complex Lie group as a subgroup $G^{\mathbb{C}} \subset S L(n, \mathbb{C})$ of $S L(n, \mathbb{C})$. Then $G^{\mathbb{C}}$ and $\mathfrak{g}^{\mathbb{C}} \subset \mathfrak{s l}(n, \mathbb{C})$ are subsets of the space $M_{n}(\mathbb{C})$ of $n \times n$ matrices, and $\mathfrak{g}^{\mathbb{C}}$ acquires a Hermitian inner product from this inclusion. ${ }^{7}$ Consider $\mathbb{P}^{1}$, equipped with the standard Kähler metric, and choose a base point in $\mathbb{P}^{1}$. Let $\lambda<-2$ and $s>(-\lambda+1) / 2$, and let $\mathcal{G}:=\operatorname{Map}_{*, \lambda+2 s+1}\left(\mathbb{P}^{1}, G^{\mathbb{C}}\right)$ be the space of maps from $\mathbb{P}^{1}$ to $G^{\mathbb{C}}$ of Sobolev class $\lambda+2 s+1$ which carry the base point in $\mathbb{P}^{1}$ to the identity in $G^{\mathbb{C}}$. We have chosen $s$ sufficiently large so that the space $\mathcal{G}$ is a Lie group, with the group structure given by pointwise multiplication. As a Hilbert manifold $\mathcal{G}$ is modelled on the Lie algebra $\operatorname{Lie}(\mathcal{G})$ given by the space $\operatorname{Lie}(\mathcal{G}):=\Omega_{*, \lambda+2 s+1}^{0}\left(\mathbb{P}^{1}, \mathfrak{g}^{\mathbb{C}}\right):=\Gamma_{*, \lambda+2 s+1}(V)$ of sections of the trivial bundle $V:=\mathbb{P}^{1} \times \mathfrak{g}^{\mathbb{C}} \longrightarrow \mathbb{P}^{1}$ of Sobolev class $\lambda+2 s+1$ which vanish at the base point of $\mathbb{P}^{1}$. For $\nu \in \mathbb{R}$, let $\Omega_{\nu}^{0,1}\left(\mathbb{P}^{1}, \mathfrak{g}^{\mathbb{C}}\right):=\Omega^{0,1}\left(\mathbb{P}^{1}, \mathfrak{g}^{\mathbb{C}}\right) \otimes H_{\nu}\left(\mathbb{P}^{1}\right)$ be the space of $\mathfrak{g}^{\mathbb{C}}$-valued $(0,1)$-forms on $\mathbb{P}^{1}$ of Sobolev class $\nu$; if $W=T^{*} \mathbb{P}^{1} \otimes \mathfrak{g}^{\mathbb{C}}$, then $\Omega_{\nu}^{0,1}\left(\mathbb{P}^{1}, \mathfrak{g}^{\mathbb{C}}\right)=\Gamma_{\nu}(W)$. The space $\Omega_{\lambda}^{0,1}\left(\mathbb{P}^{1}, \mathfrak{g}^{\mathbb{C}}\right)=\Gamma_{\lambda}(W)$ is equipped with a white noise measure $\mu_{W}$.

The group $\mathcal{G}$ acts freely on the space $\mathcal{A}_{\lambda+2 s}:=\Omega_{\lambda+2 s}^{0,1}\left(\mathbb{P}^{1}, \mathfrak{g}^{\mathbb{C}}\right)$ by the formula $g \cdot A=g A g^{-1}+\bar{\partial} g g^{-1}$ for $g \in \mathcal{G}, A \in \mathcal{A}_{\lambda+2 s}$. Let $\Delta: \Omega_{\nu+2}^{0,1}\left(\mathbb{P}^{1}, \mathfrak{g}^{\mathbb{C}}\right) \longrightarrow \Omega_{\nu}^{0,1}\left(\mathbb{P}^{1}, \mathfrak{g}^{\mathbb{C}}\right)$ denote the Laplacian.

Define the map $\mathcal{F}_{s}: \mathcal{G} \longrightarrow \Omega_{\lambda}^{0,1}\left(\mathbb{P}^{1}, \mathfrak{g}^{\mathbb{C}}\right)$ by

$$
\mathcal{F}_{s}(g)=(-\Delta+1)^{s} \bar{\partial} g g^{-1}
$$

Theorem 2. The map $\mathcal{F}_{s}$ is well-defined and differentiable. Its derivative is a Fredholm operator of index 0 . The map $\left.\delta \mathcal{F}_{s}\right|_{g}$ is an isomorphism for all $g \in \mathcal{G}$.

Therefore, for each $t>0$, the pullback measure

$$
\mu_{\mathcal{G}}^{s, t}:=\mathcal{F}_{s}^{*} \mu_{W}^{t}
$$

exists and is a $\sigma$-finite measure on $\mathcal{G}$. For $t$ sufficiently large, this measure is positive.

In the next section we will prove that the measure $\mu_{\mathcal{G}}^{s, t}$ is a Borel probability measure for all $t$; see Theorem 4

Proof. An application of Proposition 3.1 shows that $\mathcal{F}_{s}$ is well-defined and differentiable. For $g \in \mathcal{G}$, the tangent space $T \mathcal{G}_{g}$ can be identified with $\Omega_{*, \lambda+2 s+1}^{0}\left(\mathbb{P}^{1}, \mathfrak{g}^{\mathbb{C}}\right)$. The map $\mathcal{F}_{s}$ is the composite of the map $\mathcal{F}: \mathcal{G} \longrightarrow \mathcal{A}_{\lambda+2 s}$ given by $\mathcal{F}(g)=\bar{\partial} g g^{-1}$ with the isomorphism $(-\Delta+1)^{s}: \mathcal{A}_{\lambda+2 s} \longrightarrow$ $\Omega_{\lambda}^{0,1}\left(\mathbb{P}^{1}, \mathfrak{g}^{\mathbb{C}}\right)$. The map $\mathcal{F}$ gives a smooth action of $\mathcal{G}$ on $\mathcal{F}(\mathcal{G})$, which is the restriction to $\mathcal{F}(\mathcal{G})$ of the action of $\mathcal{G}$ on $\mathcal{A}_{\lambda+2 s}$. In terms of this group action, $\mathcal{F}(\mathcal{G})$ is the orbit of the point $0 \in \mathcal{A}_{\lambda+2 s}$. The derivative of $\left.\delta \mathcal{F}\right|_{e}$ at the identity $e \in \mathcal{G}$ is $\left.\delta \mathcal{F}\right|_{e}=\bar{\partial}: \Omega_{*, \lambda+2 s+1}^{0}\left(\mathbb{P}^{1}, \mathfrak{g}^{\mathbb{C}}\right) \longrightarrow \Omega_{\lambda+2 s}^{0,1}\left(\mathbb{P}^{1}, \mathfrak{g}^{\mathbb{C}}\right)$, which is an isomorphism. Therefore the derivative of $\left.\delta \mathcal{F}\right|_{g}$ is an isomorphism at any point $g \in \mathcal{G}$. Compare [3], Lemma 14.6 and (14.7).

3.3.1. The phase of the determinant of $\delta \mathcal{F}_{s}$. The derivative $\left.\delta \mathcal{F}_{s}\right|_{g}$ is given by the Fredholm operator

$$
D_{g}:=(-\Delta+1)^{s}\left(\bar{\partial}-\bar{\partial} g g^{-1}\right): \Omega_{*, \lambda+2 s+1}^{0}\left(\mathbb{P}^{1}, \mathfrak{g}^{\mathbb{C}}\right) \longrightarrow \Omega_{\lambda}^{0,1}\left(\mathbb{P}^{1}, \mathfrak{g}^{\mathbb{C}}\right) .
$$

Choose a smooth base point $g_{0} \in \mathcal{G}$. Then $\left(D_{g_{0}}\right)^{-1} D_{g}: \Omega_{*, \lambda+2 s+1}^{0}\left(\mathbb{P}^{1}, \mathfrak{g}^{\mathbb{C}}\right) \longrightarrow \Omega_{*, \lambda+2 s+1}^{0}\left(\mathbb{P}^{1}, \mathfrak{g}^{\mathbb{C}}\right)$ can be written as a perturbation of the identity

$$
\left(D_{g_{0}}\right)^{-1} D_{g}=1+K\left(g_{0}, g\right),
$$

\footnotetext{
${ }^{6}$ The appearance of regularized determinants is one reason we have chosen to work with Sobolev spaces and Hilbert manifolds rather than with Zygmund spaces and Banach manifolds. While there exist theories of determinants on Banach spaces (see e.g. 17 35]) I am not aware of a theory of regularized determinants in this context.

${ }^{7}$ The only reason for the restriction $G^{\mathbb{C}} \subset S L(n, \mathbb{C})$ is to allow products and the inverse map on the space $\mathcal{G}$ to be estimated directly using Proposition 3.1 It is not very difficult to remove this restriction; see [28].
} 
where

$$
K\left(g_{0}, g\right)=\left(\bar{\partial}-\bar{\partial} g_{0} g_{0}^{-1}\right)^{-1} \circ M\left(\bar{\partial} g_{0} g_{0}^{-1}-\bar{\partial} g g^{-1}\right) .
$$

Here for $\psi \in \Omega_{\lambda+2 s}^{0,1}\left(\mathbb{P}^{1}, \mathfrak{g}^{\mathbb{C}}\right)$, we have denoted by

$$
M(\psi): \Omega_{*, \lambda+2 s+1}^{0}\left(\mathbb{P}^{1}, \mathfrak{g}^{\mathbb{C}}\right) \longrightarrow \Omega_{\lambda+2 s}^{0,1}\left(\mathbb{P}^{1}, \mathfrak{g}^{\mathbb{C}}\right)
$$

the wedge product by $\psi$, and $\left(\bar{\partial}-\bar{\partial} g_{0} g_{0}^{-1}\right)^{-1}$ is the inverse of the isomorphism

$$
\left(\bar{\partial}-\bar{\partial} g_{0} g_{0}^{-1}\right): \Omega_{*, \lambda+2 s+1}^{0}\left(\mathbb{P}^{1}, \mathfrak{g}^{\mathbb{C}}\right) \longrightarrow \Omega_{\lambda+2 s}^{0,1}\left(\mathbb{P}^{1}, \mathfrak{g}^{\mathbb{C}}\right) .
$$

We will show that $K\left(g_{0}, \cdot\right) \in \mathcal{S}_{3}\left(\Omega_{*, \lambda+2 s+1}^{0}\left(\mathbb{P}^{1}, \mathfrak{g}^{\mathbb{C}}\right)\right)$ almost everywhere with respect to the measure $\mu_{\mathcal{G}}^{s, t}$. Since $\lambda<-2$, the white noise measure on $\Gamma_{\lambda}(W)$ is supported on $\Gamma_{\lambda+1}(W) \subset \Gamma_{\lambda}(W)$. Thus the pullback measure $\mu_{\mathcal{G}}^{s, t}=\mathcal{F}_{s}^{*} \mu_{W}^{t}$ is supported on $\operatorname{Map}_{*, \lambda+2 s+2}\left(\mathbb{P}^{1}, G^{\mathbb{C}}\right) \subset \mathcal{G}$. It follows that $\left(\bar{\partial} g_{0} g_{0}^{-1}-\bar{\partial} g g^{-1}\right) \in \Omega_{\lambda+2 s+1}^{0,1}\left(\mathbb{P}^{1}, \mathfrak{g}^{\mathbb{C}}\right)$ for $g$ lying in the complement of a set of measure zero in the measure $\mu_{\mathcal{G}}^{s, t}$.

Then, for almost all $g$,

$$
K\left(g_{0}, g\right)=\left(\bar{\partial}-\bar{\partial} g_{0} g_{0}^{-1}\right)^{-1} \circ j \circ \hat{M}\left(\bar{\partial} g_{0} g_{0}^{-1}-\bar{\partial} g g^{-1}\right) .
$$

where for $\psi \in \Omega_{\lambda+2 s+1}^{0,1}\left(\mathbb{P}^{1}, \mathfrak{g}^{\mathbb{C}}\right)$, we have denoted by

$$
\hat{M}(\psi): \Omega_{*, \lambda+2 s+1}^{0}\left(\mathbb{P}^{1}, \mathfrak{g}^{\mathbb{C}}\right) \longrightarrow \Omega_{\lambda+2 s+1}^{0,1}\left(\mathbb{P}^{1}, \mathfrak{g}^{\mathbb{C}}\right)
$$

the wedge product by $\psi$, and $j: \Omega_{\lambda+2 s+1}^{0,1}\left(\mathbb{P}^{1}, \mathfrak{g}^{\mathbb{C}}\right) \longrightarrow \Omega_{\lambda+2 s}^{0,1}\left(\mathbb{P}^{1}, \mathfrak{g}^{\mathbb{C}}\right)$ is the inclusion.

Then by Proposition [3.1, $\|\hat{M}(\psi)\| \leq\|\psi\|_{\lambda+2 s+1}$, and by the estimate (3.3),

$$
K\left(g_{0}, g\right) \in \mathcal{S}_{3}\left(\Omega_{*, \lambda+2 s+1}^{0}\left(\mathbb{P}^{1}, \mathfrak{g}^{\mathbb{C}}\right)\right)
$$

for almost all $g$. The regularized determinant $\operatorname{det}_{3}\left(1+K\left(g_{0}, g\right)\right)$ is therefore well-defined almost everywhere, and since $1+K\left(g_{0}, g\right)$ is invertible, $\operatorname{det}_{3}\left(1+K\left(g_{0}, g\right)\right)$ is nonzero for all $g$ where it is defined. Define $\Psi\left(g_{0}, \cdot\right): \mathcal{G} \longrightarrow S^{1}$ almost everywhere by

$$
\Psi\left(g_{0}, g\right)=\frac{\operatorname{det}_{3}\left(1+K\left(g_{0}, g\right)\right)}{\left|\operatorname{det}_{3}\left(1+K\left(g_{0}, g\right)\right)\right|} .
$$

Remark 3.15. The expression (3.7) for the determinant of a trace-class operator gives a conjecture for the value of the function $\Psi$. Approximate $K$ by a sequence $K_{n}$ of trace-class operators. Since $\mathcal{F}_{s}$ is a holomorphic map, we expect that morally the phase of the determinant $\operatorname{det}\left(1+K_{n}\right)$ will tend to +1 as $n \longrightarrow \infty$. In view of equation (3.7) the phase of the regularized determinant $\operatorname{det}_{3}(1+K)$ should be given morally by $\Psi=\exp \left(i \lim _{n \longrightarrow \infty} \operatorname{Im} \operatorname{tr}\left(K_{n}{ }^{2}\right)\right)$, so that we might expect $\Psi$ to be the exponential of a (possibly nonlocal) quadratic polynomial in $\bar{\partial} g g^{-1}$. This quantity is known as the anomaly in the physics literature, and often turns out to be well-defined when $K$ is taken as a limit of trace-class operators, even though $K$ is not Hilbert-Schmidt. ${ }^{8}$ See also Remark 4.7.

3.4. Three-dimensional Gauge Theory. Let $M$ be a compact, smooth Riemannian 3-manifold. Let $G$ be a compact Lie group, and choose an invariant Hermitian inner product on $\mathfrak{g}$. Choose $\lambda<-3 / 2$ and $s>(-\lambda+1 / 2) / 2$ and let $\mathcal{A}=\mathcal{A}_{\lambda+2 s+1}$ be the space of connections on the trivialized principal $G$-bundle on $M$ of Sobolev class $\lambda+2 s+1$; in the notation of Section 2, this is given by $\mathcal{A}=\Gamma_{\lambda+2 s+1}\left(T^{*} M \otimes \mathfrak{g}\right)$. For $A \in \mathcal{A}$ denote the curvature of $A$ by $F_{A}$. After a choice of a base point in $M$, connections $A$ on $M$ which satisfy the equation $F_{A}=0$ correspond by the monodromy representation to representations of $\pi_{1}(M)$ in $G$. Choose such a flat connection $A_{0}$. Let $\Omega_{\nu}^{i}(M, \mathfrak{g})=\Gamma_{\nu}\left(\Lambda^{i}\left(T^{*} M\right) \otimes \mathfrak{g}\right)$ be the space of de Rham forms on $M$ with values in $\mathfrak{g}$ of Sobolev class $\nu$. The space $\Omega_{\lambda}^{0}(M, \mathfrak{g}) \oplus \Omega_{\lambda}^{2}(M, \mathfrak{g})$ is given, in the notation of Section 2 , by $\Gamma_{\lambda}(V)$ where

\footnotetext{
${ }^{8}$ The physics literature uses a type of approximation of $K$ by a trace-class operator called dimensional regularization.
} 
$V=\mathfrak{g} \oplus\left(\Lambda^{2} T^{*} M\right) \otimes \mathfrak{g}$, and where $\lambda<-3 / 2$. It is therefore equipped with a white noise measure $\mu_{V}$.

Denote by $*$ the Hodge $*$ operator on differential forms with values in $\mathfrak{g}$ obtained from the Riemannian pairing on differential forms on $M$ and the hermitian inner product on $\mathfrak{g}$. Consider the map $\mathcal{F}_{s, A_{0}}: \mathcal{A}_{\lambda+2 s+1} \oplus \Omega_{\lambda+2 s+1}^{3}(M, \mathfrak{g}) \longrightarrow \Omega_{\lambda}^{0}(M, \mathfrak{g}) \oplus \Omega_{\lambda}^{2}(M, \mathfrak{g})$ be given by

$$
\mathcal{F}_{s, A_{0}}(A, \xi)=\left(-\Delta_{A_{0}}+1\right)^{S}\left(* d_{A_{0}} *\left(A-A_{0}\right)+F_{A}+* d_{A} * \xi\right) .
$$

Theorem 3. The map $\mathcal{F}_{s, A_{0}}$ is well-defined and differentiable. Its derivative is a Fredholm operator of index 0 . Therefore, for each $t>0$, the pullback measure

$$
\mu_{M, G}^{s, t, A_{0}}:=\mathcal{F}_{s, A_{0}}^{*} \mu_{V}^{t}
$$

exists and is a $\sigma$-finite measure on $\mathcal{A}_{\lambda+2 s+1} \oplus \Omega_{\lambda+2 s+1}^{3}(M, \mathfrak{g})$, supported on the set of regular points of $\mathcal{F}_{s, A_{0}}$.

Suppose that $A_{0}$ is chosen to be a flat connection that corresponds to an isolated conjugacy class of irreducible representations of $\pi_{1}(M)$ in $G$. Then for $t$ sufficiently large, there exists a neighborhood $W$ of $\left(A_{0}, 0\right)$ such that

$$
\infty>\mu_{M, G}^{s, t, A_{0}}(W)>0 .
$$

Proof. Again, we can use Proposition 3.1 to show that $\mathcal{F}_{s, A_{0}}$ is well-defined and differentiable. The derivative $\delta \mathcal{F}_{s, A_{0}}$ is a compact perturbation of the operator $\left(-\Delta_{A_{0}}+1\right)^{s}\left(* d_{A_{0}} *+d_{A_{0}}\right)$ : $\Omega_{\lambda+2 s+1}^{1}(M, \mathfrak{g}) \oplus \Omega_{\lambda+2 s+1}^{3}(M, \mathfrak{g}) \longrightarrow \Omega_{\lambda}^{0}(M, \mathfrak{g}) \oplus \Omega_{\lambda}^{2}(M, \mathfrak{g})$ and hence is Fredholm. Since the dimension of $M$ is odd, this operator has index zero. If $A_{0}$ is a flat connection corresponding to an isolated conjugacy class of irreducible representations of $\pi_{1}(M)$ in $G$, the kernel of $\left.\delta \mathcal{F}_{s, A_{0}}\right|_{\left(A_{0}, 0\right)}$ is zero; it follows that the cokernel is also zero, so that $\delta \mathcal{F}_{s, A_{0}}$ is an isomorphism at $\left(A_{0}, 0\right)$.

As for the Wess-Zumino-Landau-Ginzburg model we have the following conjecture, which may be viewed as giving a stochastic version of the Uhlenbeck compactness theorem for solutions of the Yang-Mills equations.

Conjecture 3.17. The measure $\mu_{M, G}^{s, t, A_{0}}$ satisfies

$$
\mu_{M, G}^{s, t, A_{0}}(1)<\infty
$$

for $t$ sufficiently large.

Suppose $A_{0}$ is a flat connection that corresponds to an isolated conjugacy class of irreducible representations of $\pi_{1}(M)$ in $G$. Conjecture 3.17 implies that for $t$ sufficiently large, the measure $\mu_{M, G}^{s, t, A_{0}}$ is (up to normalization) a Borel probability measure on $\mathcal{A}_{\lambda+2 s+1} \oplus \Omega_{\lambda+2 s+1}^{3}(M, \mathfrak{g})$.

3.4.1. The phase of the determinant of $\delta \mathcal{F}_{s, A_{0}}$. The derivative $\left.\delta \mathcal{F}_{s, A_{0}}\right|_{(A, \xi)}$ is given by the Fredholm operator

$$
D_{(A, \xi)}(\alpha, \eta)=\left(-\Delta_{A_{0}}+1\right)^{s}\left(* d_{A_{0}} * \alpha+d_{A} \alpha+* d_{A} * \eta+*[\alpha, * \xi]\right) .
$$

Suppose that $\left(A^{\prime}, 0\right)$ is a regular point of $\mathcal{F}_{s, A_{0}}$. Then $D_{\left(A^{\prime}, 0\right)}$ is invertible, and $\left(D_{\left(A^{\prime}, 0\right)}\right)^{-1} D_{(A, \xi)}=$ $1+K\left(\left(A^{\prime}, 0\right) ;(A, \xi)\right)$, where

$$
K\left(\left(A^{\prime}, 0\right) ;(A, \xi)\right): \Omega_{\lambda+2 s+1}^{1}(M, \mathfrak{g}) \oplus \Omega_{\lambda+2 s+1}^{3}(M, \mathfrak{g}) \longrightarrow \Omega_{\lambda+2 s+1}^{1}(M, \mathfrak{g}) \oplus \Omega_{\lambda+2 s+1}^{3}(M, \mathfrak{g})
$$

is given by

$$
K\left(\left(A^{\prime}, 0\right) ;(A, \xi)\right)(\alpha, \eta)=\left(D_{\left(A^{\prime}, 0\right)}\right)^{-1}\left(-\Delta_{A_{0}}+1\right)^{s}\left(\left[A-A^{\prime}, \alpha\right]+*\left[A-A^{\prime}, * \eta\right]+*[\alpha, * \xi]\right) .
$$

A similar computation to that done for the Wess-Zumino-Landau-Ginzburg and Wess-ZuminoNovikov-Witten models shows that $K\left(\left(A^{\prime}, 0\right) ;(A, \xi)\right) \in \mathcal{S}_{4}\left(\Omega_{\lambda+2 s+1}^{1}(M, \mathfrak{g}) \oplus \Omega_{\lambda+2 s+1}^{3}(M, \mathfrak{g})\right)$ for all $(A, \xi)$. Thus the regularized determinant $\operatorname{det}_{4}\left(1+K\left(\left(A^{\prime}, 0\right) ;(A, \xi)\right)\right)$ is well defined, and is nonzero 
at all regular points $(A, \xi)$ of $\mathcal{F}_{s, A_{0}}$. Let $U$ be the set of regular points of $\mathcal{F}_{s, A_{0}}$. Define $\Psi\left(\left(A^{\prime}, 0\right) ; \cdot\right)$ : $U \longrightarrow S^{1}$ by

$$
\Psi\left(\left(A^{\prime}, 0\right) ;(A, \xi)\right)=\frac{\operatorname{det}_{4}\left(1+K\left(\left(A^{\prime}, 0\right) ;(A, \xi)\right)\right)}{\left|\operatorname{det}_{4}\left(1+K\left(\left(A^{\prime}, 0\right) ;(A, \xi)\right)\right)\right|} .
$$

Then the analog of Conjecture 3.11 is the following.

Conjecture 3.19. Assume that Conjecture 3.17 holds. Define the partition function $Z_{s}^{t}(M, G)$ by $Z_{s}^{t}(M, G):=\mu_{M, G}^{s, t, A_{0}}\left(\Psi\left(\left(A^{\prime}, 0\right), \cdot\right)\right)$. Then for $t$ sufficiently large, $Z_{s}^{t}(M, G)$ is independent of $t$, and $\left|Z_{s}^{t}(M, G)\right|$ is a topological invariant of $M$. If $M$ is a homology three-sphere and $A^{\prime}=A_{0}$ is chosen to be a flat connection corresponding to a conjugacy class of isolated irreducible representations of $\pi_{1}(M)$ in $S U(2)$, then $\left|Z_{s}^{t}(M, S U(2))\right|=|\chi(M)|$ where $\chi(M)$ is the Casson invariant of $M$.

Morally this conjecture says that for $t$ large, the measure $\mu_{M, G}^{s, t, A_{0}}$ should be concentrated at the transversal solutions of the nonlinear equation $\mathcal{F}_{s, A_{0}}=0$, (or, equivalently, $\mathcal{F}_{0, A_{0}}=0$ ), which correspond to conjugacy classes of isolated irreducible representations of $\pi_{1}(M)$ in $G$. These are the representations which enter into the definition of the Casson invariant (see [1]).

Remark 3.20. As in the case of the Wess-Zumino-Novikov-Witten model, we expect that a nontrivial phase of the determinant arises from the correction terms in the regularized determinant. Since the regularized determinant appearing in (3.18) is $\operatorname{det}_{4}$, equation (3.7) leads us to expect this "anomaly" in $\Psi(A)$ to be the exponential of a cubic polynomial in $A$. It seems reasonable to conjecture that this cubic polynomial is related to the Chern-Simons invariant of $A$.

Remark 3.21. Note that if one attempts to write down the formal analog of the integral of the density (1.1), one obtains a formal expression of the type

$$
\int_{\mathcal{A}} \exp (-S(A)) \operatorname{det}\left(\delta \mathcal{F}_{s, A_{0}}\right)
$$

where $S(A)=\left|\left(-\Delta_{A_{0}}+1\right)^{s} F_{A}\right|^{2}+\ldots$

If one attempts to interpret the integral (3.22) as a perturbation of a Gaussian measure, writing $S(A)=\left|\left(-\Delta_{A_{0}}+1\right) d A\right|^{2}+I(A)$, a direct computation of Feynman diagrams shows that the expectation of $|I(A)|^{2}$ in the appropriate Gaussian measure diverges. In the language of the physics literature, the integral (3.22) is not regularized. ${ }^{9}$ Our method of constructing measures on these spaces therefore differs in an essential way from standard renormalization theory. ${ }^{10}$

\subsection{Gauge theory in two and four dimensions.}

3.5.1. Two dimensional gauge theory. Let $\Sigma$ be a Riemannian two-manifold, let $G$ be a compact Lie group. Choose an invariant Hermitian inner product on $\mathfrak{g}$. Let $\lambda<-1$, let $s>-\lambda / 2$, and let $\mathcal{A}_{\lambda+2 s+1}$ be the space of connections on the trivialized principal $G$-bundle over $\Sigma$ of Sobolev class $\lambda+2 s+1$. Let $A_{0}$ denote the product connection. Let $\Omega_{\nu}^{i}(\Sigma, \mathfrak{g})$ denote the space of de Rham forms on $\Sigma$ of Sobolev class $\nu$ with values in $\mathfrak{g}$, and let $\star$ denote the Hodge star operator on

\footnotetext{
${ }^{9}$ Naive power counting indicates that the expectation of $|I(A)|^{2}$ converges. This does not imply convergence since naive power counting is ineffective for theories with derivative interactions. One example is the expectation in the Gaussian measure with covariance $\left(-\Delta_{A_{0}}+1\right)^{-(2 s+1)}$ of the square of the cubic vertex $\mid<\left(\left(-\Delta_{A_{0}}+1\right)^{s} d A\right),((-\Delta+$ $\left.1)^{s}\left(A^{2}\right)\right)>\left.\right|^{2}$, which is divergent but for which naive power counting predicts convergence.

${ }^{10}$ Readers familiar with the physics literature might be surprised by the appearance of the non-gauge-invariant Lagrangian (3.22) and by the absence of Faddeev-Popov ghost terms. The first problem may be remedied by replacing the map $\mathcal{F}_{s, A_{0}}$ by the map $\tilde{\mathcal{F}}_{s, A_{0}}$ given by

$$
\tilde{\mathcal{F}}_{s, A_{0}}(A, \xi)=\left(-\Delta_{A}+1\right)^{s}\left(* d_{A_{0}} *\left(A-A_{0}\right)+F_{A}+* d_{A} * \xi\right)
$$

which has the same zeros. However, since this map also corresponds to a gauge-fixed theory, there is little to choose between the two maps, and we have preferred the simpler map $\mathcal{F}_{s, A_{0}}$. The absence of Faddeev-Popov ghosts is due to the cancellation of the ghost determinants between bosons and fermions in a supersymmetric theory.
} 
differential forms with values in $\mathfrak{g}$ obtained from the Riemannian pairing on differential forms and the hermitian inner product on $\mathfrak{g}$. Let $E$ be a finite-dimensional vector space of dimension $6 g-6$, and let $f: \mathcal{A}_{\lambda+2 s+1} \longrightarrow E$ be a map. Let $\mathcal{F}_{s}: \mathcal{A}_{\lambda+2 s+1} \longrightarrow \Omega_{\lambda}^{0}(\Sigma, \mathfrak{g}) \oplus \Omega_{\lambda}^{2}(\Sigma, \mathfrak{g}) \oplus E$ be given by

$$
\mathcal{F}_{s}(A)=\left(-\Delta_{A_{0}}+1\right)^{s}\left(F_{A} \oplus \star d_{A} \star\left(A-A_{0}\right)\right) \oplus f .
$$

Again, using Proposition $3.1 \mathcal{F}_{s}$ is a differentiable map of Banach manifolds, and its derivative is a Fredholm map. The index of this map depends on $f$; for $f=0$ this index is $6 g-6$, so we may expect the index of $\mathcal{F}_{s}$ for generic $f$ to be zero, and so to yield a measure on the space of connections $\mathcal{A}_{\lambda+2 s+1}$. The zeros of $\mathcal{F}_{s}$ correspond to flat connections on $\Sigma$ satisfying conditions given by the map $f$ and so, for appropriate choices of $E$ and $f$, the resulting measure may be expected to be related to intersection numbers on a moduli space of vector bundles.

3.5.2. Four dimensional gauge theory. Let $M$ be a Riemannian four-manifold, let $G$ be a compact Lie group. Let $P$ be a principal $G$-bundle over $M$. Choose an invariant Hermitian metric on $\operatorname{ad}(P)$. Let $\lambda<-2$, let $s>(-\lambda+1) / 2$, and let $\mathcal{A}_{\lambda+2 s+1}$ be the space of connections on $P$ of Sobolev class $\lambda+2 s+1$. Let $A_{0}$ be a smooth point of $\mathcal{A}_{\lambda+2 s+1}$. Let $\Omega_{\nu}^{i}(M, \operatorname{ad}(P))$ denote the space of de Rham forms on $M$ of Sobolev class $\nu$ and values in $\operatorname{ad}(P)$. Let $\star$ denote the Hodge star operator on $\operatorname{ad}(P)$-valued differential forms and let $\Omega_{\nu,+}^{2}(M, \operatorname{ad}(P))$ denote the space of selfdual de Rham forms of Sobolev class $\nu$. Let $E$ be a finite-dimensional vector space of dimension $8 p_{1}(P)-3\left(b_{2}^{+}(M)-b_{1}(M)+1\right)$, and let $f: \mathcal{A}_{\lambda+2 s+1} \longrightarrow E$ be a map. Let $\mathcal{F}_{s}: \mathcal{A}_{\lambda+2 s+1} \longrightarrow$ $\Omega_{\lambda}^{0}(M, \operatorname{ad}(P)) \oplus \Omega_{\lambda,+}^{2}(M, \operatorname{ad}(P)) \oplus E$ be given by

$$
\mathcal{F}_{s}(A)=\left(-\Delta_{A_{0}}+1\right)^{s}\left(F_{A}^{+} \oplus \star d_{A} \star\left(A-A_{0}\right)\right) \oplus f,
$$

where $F_{A}^{+}$is the self-dual part of the curvature of $A$.

Again, using Proposition 3.1 one can show that $\mathcal{F}_{s}$ is a differentiable map of Banach manifolds. Its derivative is a Fredholm map, the composition of $\left(-\Delta_{A_{0}}+1\right)^{s}$ with a compact perturbation of the standard elliptic operator $d_{A}^{+}+\star d_{A} \star: \Omega_{\lambda+2 s+1}^{1}(M, \operatorname{ad}(P)) \longrightarrow \Omega_{\lambda+2 s}^{0}(M, \operatorname{ad}(P)) \oplus$ $\Omega_{\lambda+2 s,+}^{2}(M, \operatorname{ad}(P))$.

Again, for generic $f$, we expect the index of $\delta \mathcal{F}_{s}$ to be zero, and hence to yield a measure on the space of connections $\mathcal{A}_{\lambda+2 s+1}$. The zeros of $\mathcal{F}_{s}$ correspond to self-dual connections on $M$ satisfying conditions given by the map $f$ and so, for appropriate choices of $E$ and $f$, the resulting measure may be expected to be related to Donaldson invariants of $M$.

Remark 3.26. The formal analogy between the path integrals appearing in the physics literature on gauge theory and the Mathai-Quillen formula was studied in [4].

Remark 3.27. There are other possible choices of the map $\mathcal{F}_{s}$ in this example. One alternative to the definition (3.25) is

$$
\tilde{\mathcal{F}}_{s}(A)=\left(-\Delta_{A}+1\right)^{s}\left(F_{A}^{+} \oplus \star d_{A} \star\left(A-A_{0}\right)\right) \oplus f .
$$

This choice makes contact with the work of 4 as follows. Write $\mathcal{A}$ for the space of connections on $P$ and $\mathcal{G}=\operatorname{Aut}(P)$. In the spirit of 4, the formal path integrals of 45 morally compute the Euler number of the vector bundle ${ }^{11} \mathcal{V}:=\Omega_{+}^{2}(\operatorname{ad}(P)) \times_{\mathcal{G}} \mathcal{A} \longrightarrow \mathcal{A} / \mathcal{G}$ by a formula involving the section $A \longrightarrow F_{A}^{+}$, corresponding to the map $\tilde{\mathcal{F}}_{0}$. The same computation applied to the section $A \longrightarrow\left(-\Delta_{A}+1\right)^{s}\left(F_{A}^{+}\right)$, corresponding to the map $\tilde{\mathcal{F}}_{s}$, should morally give the same result, as it is a section of the same vector bundle. In algebraic geometry there is the familiar idea of computing the number of zeros of a section of a vector bundle by finding a different section of the same vector bundle where the computation is simpler. In our setting a different choice of section takes us from the realm of formal path integrals to that of well-defined measures!

\footnotetext{
${ }^{11}$ We follow 4 and ignore the locus where the action of $\mathcal{G}$ is not free.
} 
It is possible to give a construction similar to the one we have given in this paper for measures corresponding to sections of the vector bundle $\mathcal{V} \longrightarrow \mathcal{A} / \mathcal{G}$ rather than for maps of Banach spaces. For this purpose the map $\tilde{\mathcal{F}}_{s}$ (rather than $\mathcal{F}_{s}$ ) is crucial, since it gives rise to a section of the appropriate vector bundle. Similar constructions exist for gauge theory in dimensions 2 and 3 , and indeed in higher dimensions. Details will appear elsewhere.

3.6. Quantum cohomology. Let $\Sigma$ be a Kähler two-manifold, and let $M$ be a Kähler manifold; denote by $T \Sigma$ and $T M$ the holomorphic tangent bundles of $\Sigma$ and $M$, respectively. Let $\lambda<-1$ and $s>(-\lambda+1) / 2$. Let $\operatorname{Map}_{\lambda+2 s+1}(\Sigma, M)$ be the space of maps from $\Sigma$ to $M$ of Sobolev class $\lambda+2 s+1$. Let $V$ be the vector bundle over $\operatorname{Map}_{\lambda+2 s+1}(\Sigma, M)$ whose fibre at $\phi \in \operatorname{Map}_{\lambda+2 s+1}(\Sigma, M)$ is the space $\Gamma_{\lambda}\left(\phi^{*} T^{*} M \otimes T \Sigma\right)$ of sections of $\phi^{*} T^{*} M \otimes T \Sigma$ of Sobolev class $\lambda$. Let $\mathcal{F}_{s}$ be the section of $V$ be given by

$$
\mathcal{F}_{s}(\phi)=(-\Delta+1)^{s} \bar{\partial} \phi
$$

In a sufficiently small neighborhood of any map $\phi$, the vector bundle $V$ can be trivialized as a space of maps into a vector spaces. The section $\mathcal{F}_{s}$ can then be used to push forward the white noise measure on these vector spaces to a measure on $\operatorname{Map}_{\lambda+2 s+1}(\Sigma, M)$. The analog of the MathaiQuillen formula (1.5) then differs from this measure by the usual phase of the determinant as well as by an infinite series analogous to the series $C_{k}$ appearing in (1.5); this infinite series can be shown to converge. Details will appear elsewhere.

Remark 3.29. The analogy between path integrals associated to quantum cohomology in the physics literature and the Mathai-Quillen formula associated formally to $\mathcal{F}_{0}$ was studied in [48].

\section{The Wess-Zumino-Novikov-Witten models on $\mathbb{P}^{1}$.}

In this section we make a more detailed study of the measure $\mu_{\mathcal{G}}^{s, t}$ constructed in Theorem 2 First we prove that $\mu_{\mathcal{G}}^{s, t}$ is a Borel probability measure on $\mathcal{G}$. Since $\mu_{\mathcal{G}}^{s, t}(\mathcal{G})=1$, there is no analog for $\mu_{\mathcal{G}}^{s, t}$ of Conjecture 3.11 or Conjecture 3.19] Instead we compute more subtle quantities given by expectation values of functions in the measure $\mu_{\mathcal{G}}^{s, t}$. We show that these expectation values give formulas which agree with formulas discovered by Frenkel and Zhu 12 in the context of the theory of vertex operator algebras.

4.1. The measure $\mu_{\mathcal{G}}^{s, t}$ is a probability measure. Recall that we are working with the a subgroup $G^{\mathbb{C}} \subset S L(n, \mathbb{C})$, and this identification induces a hermitian inner product on $\mathfrak{g}^{\mathbb{C}}=\operatorname{Lie}\left(G^{\mathbb{C}}\right)$. Choose $\lambda<-2$ and $s>(-\lambda+1) / 2$. Recall that $\mathcal{G}:=\operatorname{Map}_{*, \lambda+2 s+1}\left(\mathbb{P}^{1}, G^{\mathbb{C}}\right)$ is the space of based maps from $\mathbb{P}^{1}$ to $G^{\mathbb{C}}$ of Sobolev class $\lambda+2 s+1$. Let $\tilde{\mathcal{G}}:=\operatorname{Map}_{\lambda+2 s+1}\left(\mathbb{P}^{1}, G^{\mathbb{C}}\right)$ denote the space of all maps from $\mathbb{P}^{1}$ to $G^{\mathbb{C}}$ of Sobolev class $\lambda+2 s+1$; like $\mathcal{G}$, this is a Lie group with the group structure given by pointwise multiplication. Let $\mathcal{B}:=\Omega_{\lambda}^{0,1}\left(\mathbb{P}^{1}, \mathfrak{g}^{\mathbb{C}}\right)$ be the space of anti-holomorphic one forms on $\mathbb{P}^{1}$ of Sobolev class $\lambda$, and let $\mathcal{A}_{\lambda+2 s}:=\Omega_{\lambda+2 s}^{0,1}\left(\mathbb{P}^{1}, \mathfrak{g}^{\mathbb{C}}\right)$. The group $\tilde{\mathcal{G}}$ acts on $\mathcal{A}_{\lambda+2 s}$ by the gauge action

$$
g \cdot A=g A g^{-1}+\bar{\partial} g g^{-1}
$$

for $g \in \tilde{\mathcal{G}}, A \in \mathcal{A}_{\lambda+2 s}$. Recall that $\mathcal{B}=\Gamma_{\lambda}(W)$ where $W=T^{*} \mathbb{P}^{1} \otimes \mathfrak{g}^{\mathbb{C}}$, and that $\mathcal{B}$ is therefore equipped with a white noise measure $\mu_{W}$.

Recall that the map $\mathcal{F}_{s}: \mathcal{G} \longrightarrow \mathcal{B}$ is given by $\mathcal{F}_{s}(g)=(-\Delta+1)^{s} \bar{\partial} g g^{-1}$. The map $\mathcal{F}_{s}$ is the composite of the map $\mathcal{F}: \mathcal{G} \longrightarrow \mathcal{A}_{\lambda+2 s}$ given by $\mathcal{F}(g)=\bar{\partial} g g^{-1}$ with the isomorphism $(-\Delta+1)^{s}$ : $\mathcal{A}_{\lambda+2 s} \longrightarrow \mathcal{B}$. Since $\mathcal{F}: \mathcal{G} \longrightarrow \mathcal{A}_{\lambda+2 s}$ gives the orbit of $0 \in \mathcal{A}_{\lambda+2 s} \in \mathcal{A}$, and since $\mathcal{G}$ acts freely on $\mathcal{A}_{\lambda+2 s}, \mathcal{F}_{s}$ is injective. To determine the complement of the image of $\mathcal{F}_{s}$, we make use of the stratification of the space $\mathcal{A}_{\lambda+2 s}$ given in Atiyah-Bott [3], referring to the work of Shatz [36], Narasimhan-Seshadri [27, and Harder-Narasimhan [19]. See also [18, 2, 8, 17, 31. 
Proposition 4.1. The image of $\mathcal{F}_{s}$ consists of the complement of a countable union of smooth, locally closed subvarieties $V_{i}$ of $\mathcal{B}$. Each of these subvarieties is of the form $V_{i}=(-\Delta+1)^{s} \tilde{\mathcal{G}} \cdot A_{i}$ where $A_{i}$ is a smooth element of $\Omega^{0,1}\left(\mathbb{P}^{1}, \mathfrak{g}^{\mathbb{C}}\right)$. The subvariety $V_{i}$ has positive codimension in $\mathcal{B}$, and the normals to $V_{i}$ at $(-\Delta+1)^{s} A_{i}$ are smooth.

Proof. Since the map $(-\Delta+1)^{s}: \mathcal{A}_{\lambda+2 s} \longrightarrow \mathcal{B}$ is an isomorphism, it is enough to prove an analogous result for the image of the map $\mathcal{F}: \mathcal{G} \longrightarrow \mathcal{A}_{\lambda+2 s}$. This the orbit of the point $0 \in \mathcal{A}_{\lambda+2 s}$ under the action of $\tilde{\mathcal{G}}$.

The results of [3] show that the space $\mathcal{A}_{\lambda+2 s}$ can be stratified as $\mathcal{A}_{\lambda+2 s}=\coprod_{\mu} \mathcal{A}_{(\mu)}$, where the $\mathcal{A}_{(\mu)}$ are a countable family of $\tilde{\mathcal{G}}$ orbits which are smooth locally closed subvarieties of $\mathcal{A}_{\lambda+2 s}$, each of finite codimension in $\mathcal{A}_{\lambda+2 s}$. On $\mathbb{P}^{1}$ these strata are given by $\tilde{\mathcal{G}}$-orbits of a countable collection of elements $A_{\mu} \in \mathcal{A}_{\lambda+2 s}$. (See [3], Section 14, pp. 608-609, 7], or 31], Theorem 2 and its Corollary).

The $\tilde{\mathcal{G}}$-orbit of the trivial connection $A_{0}$ corresponding under our identification to $0 \in \Omega_{\lambda+2 s}^{0,1}\left(\mathbb{P}^{1}, \mathfrak{g}^{\mathbb{C}}\right)$ is the image of the map $\mathcal{F}$. We denote the corresponding stratum by $\mathcal{A}_{(0)}$.

Each stratum $\mathcal{A}_{(\mu)}$ contains a smooth point by Lemma 14.8 of $\left[3\right.$. The normals to $\mathcal{A}_{(\mu)}$ at a smooth point $A \in \mathcal{A}_{(\mu)}$ are given by the kernel of the adjoint $D_{A}^{*}$ of the elliptic operator $D_{A}:=$ $\bar{\partial}+A: \Omega^{0}\left(\mathbb{P}^{1}, \mathfrak{g}^{\mathbb{C}}\right) \longrightarrow \Omega^{0,1}\left(\mathbb{P}^{1}, \mathfrak{g}^{\mathbb{C}}\right)$, and are therefore smooth (see [3], Section 14, p. 608). The kernel of $D_{A}^{*}$ at smooth points was computed in [3], where it is shown to be zero for the stratum $\mathcal{A}_{(0)}$ and nonzero for every other stratum; see Proposition 5.4, (5.10), (7.15), and (10.7) of [3].

In the Yang-Mills context each of these strata is the orbit under the complexified gauge group of a solution of the Yang-Mills equation. The minimum of the Yang-Mills functional, given by the trivial connection, has the open orbit $\mathcal{F}(\mathcal{G})$.

We therefore obtain the following result.

Proposition 4.2. The map $\mathcal{F}_{s}$ is a diffeomorphism of $\mathcal{G}$ with the complement of a set of measure zero with respect to the white noise measure $\mu_{W}^{t}$ on $\mathcal{B}$ for any $t$.

Proof. Since the map $(-\Delta+1)^{s}: \mathcal{A}_{\lambda+2 s} \longrightarrow \mathcal{B}$ is an isomorphism, it suffices to show that the subvariety $Z_{i} \subset \mathcal{A}_{\lambda+2 s}$ given by $Z_{i}=\tilde{\mathcal{G}} \cdot A_{i}$ has measure zero with respect to the Gaussian measure $\mu_{W, s}^{t}$ on the space $\mathcal{A}_{\lambda+2 s}=\Omega_{\lambda+2 s}^{0,1}\left(\mathbb{P}^{1}, \mathfrak{g}^{\mathbb{C}}\right)$.

Let $a \in Z_{i}$. By the inverse function theorem, we can find $\epsilon>0$ such that the orthogonal projection $\left.B_{\epsilon}(a) \cap Z_{i} \longrightarrow T Z_{i}\right|_{q}$ is an isomorphism for all $q \in B_{\epsilon}(a) .{ }^{12}$

Let $U=B_{\epsilon / 2}(a)$. Since $Z_{i}=\tilde{\mathcal{G}} \cdot A_{i}$, the point $a$ is given by $a=g \cdot A_{i}$ for some $g \in \tilde{\mathcal{G}}$. Let $g^{\prime} \in \tilde{\mathcal{G}}$ be a smooth element of $\tilde{\mathcal{G}}$ sufficiently close to $g$ so that $g^{\prime} \cdot A_{i} \in U \cap Z_{i}$. Then $p:=g^{\prime} \cdot A_{i}$ is smooth. The choice of $\epsilon$ is such that the orthogonal projection $\left.B_{\epsilon}(a) \cap Z_{i} \longrightarrow T Z_{i}\right|_{p}$ is an isomorphism.

Let $\left.\nu \in T Z_{i}\right|_{p} ^{\perp}$. Then $\nu$ is smooth, and there exists $\alpha>0$ so that if $\delta, \delta^{\prime} \in(0, \alpha)$ and $\delta \neq \delta^{\prime}$, then

$$
\left(U \cap Z_{i}+\delta \nu\right) \cap\left(U \cap Z_{i}+\delta^{\prime} \nu\right)=\emptyset .
$$

Suppose that $\mu_{W, s}^{t}\left(U \cap Z_{i}\right)=\eta>0$. By the Cameron-Martin formula (Proposition 2.2), the Radon-Nikodym derivative $d\left(T_{c \nu}\right)_{*} \mu_{W, s}^{t} / d \mu_{W, s}^{t}$ is bounded from below for $q \in U$ and $c>0$ by

$\frac{d\left(T_{c \nu}\right)_{*} \mu_{W, s}^{t}}{d \mu_{W, s}^{t}}(q)=\exp \left(c t^{2}<(-\Delta+1)^{2 s} \nu, q>-\frac{1}{2} c^{2} t^{2}\|\nu\|_{2 s}^{2}\right) \geq \exp \left(-c t^{2}\|\nu\|_{-\lambda+2 s}\|q\|_{\lambda+2 s}-\frac{1}{2} c^{2} t^{2}\|\nu\|_{2 s}^{2}\right)$.

\footnotetext{
${ }^{12}$ Given a subspace $T \subset \mathcal{A}_{\lambda+2 s}$, denote by $\pi_{T}: \mathcal{A}_{\lambda+2 s} \longrightarrow T$ the orthogonal projection onto $T$. Define $f:$ $Z_{i} \times\left. Z_{i} \longrightarrow T Z_{i}\right|_{a} \times\left. T Z_{i}\right|_{a}$ by

$$
f(p, q)=\left(\pi_{\left.T Z_{i}\right|_{a}} \circ \pi_{\left.T Z_{i}\right|_{q}} p, \pi_{\left.T Z_{i}\right|_{a}} q\right) .
$$

Then $\delta f_{(a, a)}$ is an isomorphism. By the inverse function theorem, $f$ is a diffeomorphism in a neighborhood of $(a, a)$. It follows that the orthogonal projection $\left.\pi_{\left.T Z_{i}\right|_{q}}\right|_{Z_{i}}$ is an isomorphism near $a$ for $q$ near $a$.
} 
Since for $q \in U,\|q\|_{\lambda+2 s}<\|a\|_{\lambda+2 s}+\epsilon$, there exists $\beta>0$ such that for all $q \in U$ and all $c \in(0, \beta)$,

$$
\frac{d\left(T_{c \nu}\right)_{*} \mu_{W, s}^{t}}{d \mu_{W, s}^{t}}(q)>\frac{1}{2} .
$$

Then if $c \in(0, \beta)$,

Let $\gamma=\min \{\alpha, \beta\}$. Then

$$
\mu_{W, s}^{t}\left(U \cap Z_{i}+c \nu\right)>\frac{1}{2} \eta
$$

$$
\mu_{W, s}^{t}\left(\coprod_{n=1}^{\infty}\left(Z_{i} \cap U+\frac{\gamma}{2^{n}} \nu\right)\right)=\infty,
$$

which is impossible. So it must be that $\mu_{W, s}^{t}\left(U \cap Z_{i}\right)=0$.

Thus every point $p \in Z_{i}$ has a neighborhood in $Z_{i}$ of measure zero. Since the space $\mathcal{A}_{\lambda+2 s}$ has a countable basis for its topology, so does the subspace $Z_{i}$. Thus $Z_{i}$ is Lindelöf, and can be covered by countably many open sets of measure zero. It follows that $\mu_{W, s}^{t}\left(Z_{i}\right)=0$.

As a corollary we get the following theorem.

Theorem 4. The measure $\mu_{\mathcal{G}}^{s, t}$ is a Borel probability measure on $\mathcal{G}$ for all $t$.

4.2. The Frenkel-Zhu formula. We now focus on the case $G=S U(n)$, so that $G^{\mathbb{C}}=S L(n, \mathbb{C})$. To compare expectations in the measure $\mu_{\mathcal{G}}^{s, t}$ to results arising from the theory of vertex operator algebras, we introduce some functions on $\mathcal{G}$. Recall that $\Omega_{\lambda+2 s}^{0,1}\left(\mathbb{P}^{1}, \mathfrak{g}^{\mathbb{C}}\right)=\Gamma_{\lambda+2 s}(W)$ where $W=$ $T^{*} \mathbb{P}^{1} \otimes \mathfrak{g}^{\mathbb{C}}$. We have equipped $\mathbb{P}^{1}$ with a Kahler metric, and the Lie algebra $\mathfrak{g}^{\mathbb{C}}$ comes with an inner product given by $(a, b) \longrightarrow \operatorname{tr} a^{*} b$. Let us denote by $\star: \Omega^{0,1}\left(\mathbb{P}^{1}\right) \longrightarrow \Omega^{1,0}\left(\mathbb{P}^{1}\right)$ the Hodge star operator. Also, let $\mathcal{C}_{z}:\left.T^{*} \mathbb{P}^{1}\right|_{z} \otimes\left(\left.T^{*} \mathbb{P}^{1}\right|_{z}\right)^{*} \longrightarrow \mathbb{C}$ denote the contraction.

The inner product on $\mathfrak{g}^{\mathbb{C}}$ along with the Riemannian structure on $\mathbb{P}^{1}$ give rise to a pairing

$$
\langle,\rangle: \Omega_{\lambda+2 s}^{0,1}\left(\mathbb{P}^{1}, \mathfrak{g}^{\mathbb{C}}\right) \times \Omega_{\lambda+2 s}^{0,1}\left(\mathbb{P}^{1}, \mathfrak{g}^{\mathbb{C}}\right) \longrightarrow H_{\lambda+2 s}\left(\mathbb{P}^{1}\right) ;
$$

here we have used Proposition 3.1 to see that the inner product of elements in $\Omega_{\lambda+2 s}^{0,1}\left(\mathbb{P}^{1}, \mathfrak{g}^{\mathbb{C}}\right)$ is in $H_{\lambda+2 s}\left(\mathbb{P}^{1}\right)$.

Similarly if we are given any hermitian matrix $x$ we obtain from the pairing on $\mathfrak{g}^{\mathbb{C}}$ given by $(a, b) \longrightarrow \operatorname{tr} a^{*} x b$ a pairing

$$
\langle,\rangle_{x}: \Omega_{\lambda+2 s}^{0,1}\left(\mathbb{P}^{1}, \mathfrak{g}^{\mathbb{C}}\right) \times \Omega_{\lambda+2 s}^{0,1}\left(\mathbb{P}^{1}, \mathfrak{g}^{\mathbb{C}}\right) \longrightarrow H_{\lambda+2 s}\left(\mathbb{P}^{1}\right) .
$$

Let $x \in \mathfrak{g}$ be a hermitian matrix and let $z \in \mathbb{P}^{1}$. We define a function $\hat{F}_{x, z}: \mathcal{G} \longrightarrow \mathbb{R}$ by

$$
\hat{F}_{x, z}(g)=\left\langle\bar{\partial} g g^{-1}, \bar{\partial} g g^{-1}\right\rangle_{x}(z)
$$

We also define $F_{x, z}: \mathcal{G} \longrightarrow \mathbb{R}$ by

$$
F_{x, z}:=\hat{F}_{x, z}-\mu_{\mathcal{G}}^{s, 1}\left(\hat{F}_{x, z}\right) .
$$

Similarly, let $v \in \Omega^{0,1}\left(\mathbb{P}^{1}, \mathfrak{g}^{\mathbb{C}}\right)$, let $z \in \mathbb{P}^{1}$, and define $f_{v, z}: \mathcal{G} \longrightarrow \mathbb{C}$ by

$$
f_{v, z}(g)=\left\langle v, \bar{\partial} g g^{-1}\right\rangle(z) \text {. }
$$

We recall some definitions from [12]. Given a finite set $A$, let $\mathcal{P}_{1}(A)$ denote the set of partitions of $A$ into cycles and chains containing precisely one chain. Now let $A=\{1, \ldots, n\}$. Given $\alpha \in \mathcal{P}_{1}(A)$, let us write $\alpha$ explicitly as a collection of $k$ cycles and one chain of length $m$ :

$$
\alpha=\left\{\left(a(1,1), \ldots, a\left(1, j_{1}\right)\right), \ldots,\left(a(k, 1), \ldots, a\left(k, j_{k}\right)\right),[b(1), \ldots, b(m)]\right\} .
$$

Let $C_{s}\left(z, z^{\prime}\right)$ denote the Green's kernel of the self-adjoint operator $(-\Delta+1)^{2 s}$ on $\Omega_{0}^{0,1}\left(\mathbb{P}^{1}\right)$. Then $\left.C_{s}\left(z, z^{\prime}\right) \in\left(\left.T^{*} \mathbb{P}^{1}\right|_{z^{\prime}}\right)^{*} \otimes T^{*} \mathbb{P}^{1}\right|_{z}$. 
Let $v, v^{\prime} \in \Omega^{0,1}\left(\mathbb{P}^{1}, \mathfrak{g}^{\mathbb{C}}\right)$. We may write $v=\phi \xi, v^{\prime}=\phi^{\prime} \xi^{\prime}$ where $\phi, \phi^{\prime} \in C^{\infty}\left(\mathbb{P}^{1}, \mathfrak{g}^{\mathbb{C}}\right)$ and $\xi, \xi^{\prime} \in$ $\Omega^{0,1}\left(\mathbb{P}^{1}\right)$.

Let $x_{1}, \ldots, x_{n} \in \mathfrak{g}$, and let $z_{1}, \ldots, z_{n}, z, w \in \mathbb{P}^{1}$. We define

$$
\begin{gathered}
f_{v, v^{\prime}, \alpha ; s}\left(z, w ; z_{1}, \ldots, z_{n}\right)=\prod_{p=1}^{k} \operatorname{tr}\left(x_{a(p, 1)} \ldots x_{a\left(p, j_{p}\right)}\right) \times \operatorname{tr}\left(\phi(z)^{*} x_{b(1)} \ldots x_{b(m)} \phi^{\prime}(w)\right) \times \\
\prod_{p=1}^{k} \mathcal{C}_{z_{a(p, 1)}} \ldots \mathcal{C}_{z_{a\left(p, j_{p}\right)}}\left(C_{s}\left(z_{a(p, 1)}, z_{a(p, 2)}\right) \otimes \cdots \otimes C_{s}\left(z_{a\left(p, j_{p}\right)}, z_{a(p, 1)}\right)\right) \times \\
\mathcal{C}_{z} \mathcal{C}_{z_{b(1)}} \ldots \mathcal{C}_{z_{b(m)}} \mathcal{C}_{w}\left((\star \xi)(z) \otimes C_{s}\left(z, z_{b(1)}\right) \otimes C_{s}\left(z_{b(1)}, z_{b(2)}\right) \otimes \ldots C_{s}\left(z_{b(m-1)}, z_{b(m)}\right) \otimes C_{s}\left(z_{b(m)}, w\right) \otimes \xi^{\prime}(w)\right)
\end{gathered}
$$

Then we have the following result.

Theorem 5. Let $x_{1}, \ldots, x_{n} \in \mathfrak{g}$ and let $z_{1}, \ldots, z_{n}, z, w \in \mathbb{P}^{1}$. Let $v, v^{\prime} \in \Omega^{0,1}\left(\mathbb{P}^{1}, \mathfrak{g}^{\mathbb{C}}\right)$. Then

$$
\mu_{\mathcal{G}}^{s, 1}\left(\bar{f}_{v, z} f_{v^{\prime}, w} F_{x_{1}, z_{1}} \ldots F_{x_{n}, z_{n}}\right)=\sum_{\alpha \in \mathcal{P}_{1}(A)} f_{v, v^{\prime}, \alpha ; s}\left(z, w ; z_{1}, \ldots, z_{n}\right) .
$$

Proof. By Theorem 4.2 we may transfer the computation to the space $\mathcal{B}$, equipped with its white noise measure $\mu_{W}$, using the map $\mathcal{F}_{s}$. For any $x \in \mathfrak{g}, z \in \mathbb{P}^{1}$, we have $F_{x, z}=\mathcal{F}_{s}^{*} H_{x, z}$ where $H_{x, z}: \mathcal{G} \longrightarrow \mathbb{C}$ is given by $H_{x, z}:=\hat{H}_{x, z}-\mu_{W}\left(\hat{H}_{x, z}\right)$, and where $\hat{H}_{x, z}: \mathcal{B} \longrightarrow \mathbb{C}$ is given by

$$
\hat{H}_{x, z}(A)=\left\langle(-\Delta+1)^{-s} A,(-\Delta+1)^{-s} A\right\rangle_{x}(z)
$$

for $A \in \mathcal{B}$.

Similarly, if $v \in \Omega^{0,1}\left(\mathbb{P}^{1}, \mathfrak{g}^{\mathbb{C}}\right)$ and $z \in \mathbb{P}^{1}, f_{v, z}=\mathcal{F}_{s}^{*} h_{v, z}$ where $h_{v, z}: \mathcal{B} \longrightarrow \mathbb{C}$ is given by

$$
h_{v, z}(A)=\left\langle v,(-\Delta+1)^{-s} A\right\rangle(z) \text {. }
$$

Thus

$$
\mu_{\mathcal{G}}^{s, 1}\left(\bar{f}_{v, z} f_{v^{\prime}, w} F_{x_{1}, z_{1}} \ldots F_{x_{n}, z_{n}}\right)=\mu_{W}\left(\bar{h}_{v, z} h_{v^{\prime}, w} H_{x_{1}, z_{1}} \ldots H_{x_{n}, z_{n}}\right) .
$$

The expectation $\mu_{W}\left(\bar{h}_{v, z} h_{v^{\prime}, w} H_{x_{1}, z_{1}} \ldots H_{x_{n}, z_{n}}\right)$, like that of any polynomial, can be computed by using formula (2.1). Let $\phi_{i} \in \Omega_{-\lambda}^{0,1}\left(\mathbb{P}^{1}, \mathfrak{g}^{\mathbb{C}}\right), i=1, \ldots, 2 k$. Let $t_{1}, \ldots, t_{2 k} \in \mathbb{C}$ and let $\zeta:=\sum_{i} t_{i} \phi_{i}$. For $i=1, \ldots, 2 k$, let $L_{\phi_{i}}: \mathcal{B} \longrightarrow \mathbb{C}$ be given by $L_{\phi_{i}}(A)=<\phi_{i}, A>$. Applying formula (2.1) to $E_{\zeta}$ gives a generating function $\mu_{W}\left(E_{\zeta}\right)$ for the expectations of all polynomials in the $L_{\phi_{i}}$. Explicitly, we have Wick's theorem: Let $\mathcal{Q}_{2 k}$ be the set of partitions of the finite set $\{1, \ldots, 2 k\}$ into a collection of pairs $(a, b)$ where $a \in\{1, \ldots, k\}$ and $b \in\{k+1, \ldots, 2 k\}$. Then

$$
\mu_{W}\left(\prod_{i=1}^{k} L_{\phi_{i}} \prod_{i=k+1}^{2 k} \bar{L}_{\phi_{i}}\right)=\sum_{q \in \mathcal{Q}_{2 k}} \prod_{(a, b) \in q}<\phi_{a}, \phi_{b}>.
$$

Applying equation (4.6) to the polynomial $\bar{h}_{v, z} h_{v^{\prime}, w} H_{x_{1}, z_{1}} \ldots H_{x_{n}, z_{n}}$ gives (4.4).

The formula on the right hand side of equation (4.4), when evaluated at $s=0$, is (up to a constant) the formula given in Theorem 2.3.1 of Frenkel and Zhu [12, who work with the theory of vertex operator algebras. In our context we have obtained a type of analytic continuation of this formula from a commutative algebra of functions on $\mathcal{G}$.

Remark 4.7. The Wess-Zumino-Novikov-Witten model as it appears in the physics literature differs from the formal path integral given by using the formula (1.1) for the map $\mathcal{F}_{0}$ in two ways. First, the maps appearing in the Wess-Zumino-Novikov-Witten path integral in the physics literature are maps with values in the compact Lie group $G$ (rather than $G^{\mathbb{C}}$ ); second, the partition function is given by the integral with respect to this measure of a power of a certain function $\Phi$. 
Regarding the first issue, the work of Wendt [42] shows that the quantization of the loop group $L G$ formally corresponds to a path integral over a space of maps into $G^{\mathbb{C}}$, so that a path integral over maps into $G^{\mathbb{C}}$ may be the correct model for the study of the quantization of $L G$. Another way of seeing this is to repeat our construction for manifolds with boundary, using the results of Donaldson [9] to substitute for Proposition 4.1. Details will appear elsewhere.

We now discuss the role of the function $\Phi$. Recall that the analogy with finite dimensions leads us to believe that one should compute expectations in the measure $\mu_{\mathcal{G}}^{s, 1}(\Psi \cdot)$, where $\Psi$ is the phase of the regularized determinant calculated in (3.14). In fact the physics literature refers to a calculation of an expectation of the type $\mu\left(\Psi \Phi^{k} \cdot\right)$, where $k \in \mathbb{Z}, \mu$ is a morally a measure on the space $\mathcal{G}_{u}:=\operatorname{Map}_{*}\left(\mathbb{P}^{1}, G\right)$, and the function $\Phi: \mathcal{G}_{u} \longrightarrow S^{1}$ is given by $\Phi=\exp \left(\frac{i}{12 \pi} \int_{B} \operatorname{tr}\left(\gamma^{-1} d \gamma\right)^{3}\right)$, where $B$ is a three-manifold with $\partial B=\mathbb{P}^{1}$, and $\gamma$ is an arbitrary extension of $g$ to $B$.

The function $\Phi$ can be interpreted as a flat section of the restriction to the orbit $\mathcal{G}_{u} \cdot 0 \subset \mathcal{A}_{\lambda+2 s}$ of a hermitian line bundle with connection $\mathcal{L} \longrightarrow \mathcal{A}_{\lambda+2 s}$ [33] on the space $\mathcal{A}_{\lambda+2 s}$. This section cannot be extended to $\mathcal{G} \cdot 0$ as a flat section since $\mathcal{L}$ is not flat on $\mathcal{G} \cdot 0$. However, since the space $\mathcal{A}_{\lambda+2 s}$ is affine, one can define a function $\hat{\Phi}: \mathcal{A}_{\lambda+2 s} \longrightarrow S^{1}$ by choosing a base point $A_{0} \in \mathcal{A}_{\lambda+2 s}$ and letting $\hat{\Phi}(A)$ be the holonomy of the connection on $\mathcal{L}$ along the straight line path from $A_{0}$ to $A$. Now the determinant line bundle [30, on $\mathcal{A}_{\lambda+2 s}$ is given by $\mathcal{L}^{h}$, where $h$ is the dual Coxeter number of $G$, and the curvature of this line bundle is given [30] by a quadratic Kähler potential. This means it is reasonable to expect that the function $\hat{\Phi}$ is a the exponential of the pull back to $\mathcal{G}$ of a quadratic function on $\mathcal{A}_{\lambda+2 s}$. In this picture the phase $\Psi$ of the determinant appearing in (3.14) should be replaced by the section $\hat{\Phi}^{h}$ of the determinant line bundle $\mathcal{L}^{h}$, which arises from a method of regularizing determinants different from that of [37]. A result similar to that of

Theorem 5 holds also if the measure $\mu_{\mathcal{G}}^{s, 1}$ is replaced by the complex valued measure $\mu_{\mathcal{G}}^{s, 1}\left(\hat{\Phi}^{k+h} \cdot\right)$ where $\hat{\Phi}(g)=\exp \left(i Q\left(\bar{\partial} g g^{-1}\right)\right)$ and $Q$ is any quadratic function on $\mathcal{A}_{\lambda+2 s}$.

It is reasonable to expect that the function $\Psi: \mathcal{A}_{\lambda+2 s} \longrightarrow S^{1}$ defined in equation (3.14) is also a section of $\mathcal{L}^{h}$. Note that if this is the case, the ideas of Remark 3.15 would again lead us to believe that $\Psi$ should be of the form $\Psi(g)=\exp \left(i Q\left(\bar{\partial} g g^{-1}\right)\right)$ where $Q$ is a (possibly nonlocal) quadratic function on $\mathcal{A}_{\lambda+2 s}$.

\section{Conclusions}

5.1. Analytic continuation. The measures constructed on Banach spaces in the Wess-ZuminoNovikov-Witten model give analytic continuations of correlation functions arising in the associated theory of vertex operator algebras. This is reminiscent of the case of correlation functions in Minkowskian quantum field theory, which arise as analytic continuations of Euclidean correlation functions which can be computed by Euclidean path integrals.

5.2. Higher derivatives. The path integrals in the examples studied above correspond to partial differential equations of higher order than those arising in the problems studied by the physicists. One may wonder if Lagrangians with higher derivatives may be useful as models in physics.

5.3. Supersymmetry. Our construction uses supersymmetry in an essential way. From this point of view the supersymmetric models are more fundamental than the bosonic models; indeed one could try to construct the bosonic model by showing that the inverse of an appropriate determinant is integrable with respect to the supersymmetric measure.

5.4. Quantum field measures are not perturbations of Gaussian measures. The measures appearing in our work are not in general perturbations of Gaussian measures; they are locally pushforwards of such measures. In particular, factors of the type $(-\Delta+1)^{s}$, though they resemble dimensional regularization, may not suffice to regularize the theory. In effect the singularities of quantum field theory fall into two categories: Singularities arising from attempting to define nonlinear functions on spaces of distributions, which disappear when higher-order partial differential 
equations are considered, and singularities arising from the fact that a pullback measure may not be absolutely continuous with respect to Gaussian measure (as the Cameron-Martin theorem shows is the case for translations), which should not be removed (see Remark 3.21). The physics literature makes no distinction between these two types of singularities. Therefore the predictions of standard renormalization theory, that gauge theory in dimensions higher than four should not exist, and that sigma-models should exist only for manifolds of positive curvature, may not apply to supersymmetric models constructed by our methods. In fact gauge theory in six dimensions [34, 10, 41] and eight dimensions [6] has been studied in the physics literature, and corresponds to mathematical results, as is the case for quantum cohomology for manifolds which do not have positive curvature.

5.5. Anomalies. The physics literature assigns a special role to the phase of the determinant of the tangential operator $\delta \mathcal{F}_{s}$. The invariants of maps in the finite dimensional case involve the phase of the determinant of the tangential operator. But in infinite dimensions, it is only the regularized determinant that can be defined. The construction of this determinant involves arbitrary choices (in our case, a choice of a base point) and its phase may therefore not lead to a topological invariant, or else fail to have the same symmetries as the map $\mathcal{F}_{s}$. In the physics literature the appearance of such an "anomaly" is held to preclude the existence of a well-defined theory. The fact that we obtain a canonical construction of a measure but that the construction of the phase of the determinant involved arbitrary choices is in line with physicists' intuition, which singled out the importance of these anomaly terms.

5.6. Noncommutative and commutative algebras. Comparison with the Frenkel-Zhu formula shows that a result arising from a noncommutative vertex operator algebra can be viewed in terms of the path integral as coming from a commutative algebra. The original Feynman-Kac formula is another reflection of this principle. It may be hoped that a better understanding of the role of path integrals may be helpful in interpreting algebraic structures arising in mathematics motivated by quantum field theory. For example, a construction of the Wess-Zumino-Novikov-Witten model in genus one should give a proof of the Kac character formula 22] as adumbrated in Wendt [42].

5.7. Equivariance. In the presence of a group action it should be possible to define equivariant versions of the measures $\mathcal{F}_{s}{ }^{*} \mu$; this will involve a convergent infinite series of the type appearing in (1.5). The equivariant integral should satisfy a localization formula as in finite dimensions. In the case of the Wess-Zumino-Novikov-Witten model the equivariant extension was written down by Wendt [42]; it is closely related to the coset models studied by Gawedzki and Kupiainen [13].

5.8. Interpretation of the partition function. It would be interesting to have a geometric interpretation of finite dimensional analogs of the partition functions $\mathcal{F}_{s}^{*} \mu\left(\Psi^{k}\right)$ analogous to the interpretation of $\mathcal{F}_{s}^{*} \mu(\Psi)$ as a degree in formula (1.2).

Acknowledgments: I am grateful to I. Frenkel, V. Guillemin, A. Jaffe, V. Jones, S. Sternberg, D. Stroock, S. T. Yau, and G. Zuckerman for many helpful comments, discussions and suggestions. I would particularly like to thank I. Frenkel for bringing the work of Frenkel-Zhu [12] and Wendt 42 to my attention, D. Stroock for his generosity in answering questions on the analytical aspects of this work, and V. Guillemin for many productive suggestions and corrections, and encouragement during the course of this work.

After completing this work I received a copy of the work of C. Taubes [40, which explores a different way of constructing quantum field measures on some of the spaces we have considered. I hope that a combination of our methods with those of [40] will be useful in reviving interest in mathematical approaches to quantum field theory. 


\section{REFERENCES}

[1] S. Akbulut and J. McCarthy. Casson's Invariant for Oriented Homology 3-Spheres: An Exposition. Princeton University Press, 1990.

[2] M. Atiyah. Proc. Lond. Math. Soc. 7, 414-452 (1957)

[3] M. Atiyah and R. Bott. Philos. Trans. Roy. Soc. London Ser. A 308 (1983), no. 1505, 523-615

[4] M. Atiyah and L. Jeffrey. J. Geom. Phys. 7, 119-136 (1990)

[5] M. Atiyah and I. Singer. Proc. Nat. Acad. Sci. USA 81, 2597-2600 (1984)

[6] L. Baulieu, H. Kanno, I. Singer. Commun. Math. Phys. 194, 149-175 (1998)

[7] G. Daskalopoulos. J. Differential Geom. 36 (1992), no. 3, 699-746

[8] S. Donaldson. J. Differential Geom. 18 (1983), no. 2, 269-277.

[9] S. Donaldson. J. Geom. Phys. 8, 89-122 (1992)

[10] S. Donaldson, R. Thomas. Gauge theory in higher dimensions. The geometric universe (Oxford, 1996), 31-47, Oxford Univ. Press, Oxford, 1998.

[11] N. Dunford and J. Schwartz, Linear Operators, Part II: Spectral Theory. New York: Interscience, 1963.

[12] I. Frenkel, Y. Zhu. Duke Math. J. 66, 123-168 (1992)

[13] K. Gawedzki, A. Kupiainen. Phys. Lett. B 215, 119-123 (1988)

[14] E. Getzler. J. Func. An. 68, 388-403 (1986)

[15] E. Getzler. J. Func. An. 74, 121-138 (1987)

[16] J. Glimm, A. Jaffe. Quantum Physics. Springer, 1987.

[17] A. Grothendieck. Bull. Soc. Math. Fr. 84, 319-364 (1956)

[18] A. Grothendieck, Am. J. Math. 79, 121-138 (1957)

[19] G. Harder and M. S. Narasimhan. Math. Annln. 212, 215-248 (1975)

[20] L. Hormander. Lectures on Nonlinear Hyperbolic Differential Equations. Springer, 1997.

[21] A. Jaffe, A. Lesniewski, J. Weitsman. Commun. Math. Phys. 112, 75-88 (1987)

[22] V. Kac. Infinite Dimensional Lie Algebras. Cambridge, 1990.

[23] H.-H. Kuo. Trans. Amer. Math. Soc. 159, 57-78 (1971)

[24] S. Kusuoka. J. Fac. Sci. Univ. Tokyo Sect. IA Math. 29, 79-95 (1982)

[25] S. Lang. Differential and Riemannian Manifolds. Springer, 1996.

[26] V. Mathai and D. Quillen. Topology 25, 85-110 (1986)

[27] M. Narasimhan, C. Seshadri. Ann. Math. 82, 540-565 (1965)

[28] R. Palais. Foundations of Global Nonlinear Analysis. Benjamin, 1965.

[29] H. Poincaré. Acta Math. 33 (1910), 57-86

[30] D. Quillen. Funk. Analiz Prilozh. 19, 37-41 (1985)

[31] J. Rade. J. Reine Angew. Math. 431 (1992), 123-163

[32] R. Ramer. J. Funct. Anal. 15, 166-187 (1974)

[33] T. Ramadas, I. Singer, J. Weitsman. Commun. Math. Phys. 126, 409-420 (1989)

[34] L. Rozansky, E. Witten. Selecta Math. 3, 401-458 (1997)

[35] A. Ruston. Fredholm Theory in Banach Spaces. Cambridge University Press, 1986.

[36] S. Shatz, Compositio Math. 35, 163-187 (1977)

[37] B. Simon, Trace Ideals and Their Applications. Cambridge University Press, 1979.

[38] E. Stein. Singular Integrals and Differentiability Properties of Functions. Princeton, 1970.

[39] D. Stroock, S. R. S. Varadhan. Multidimensional Diffusion Processes. Springer, 1979.

[40] C. Taubes. Constructions of measures and quantum field theories on mapping spaces. Preprint.

[41] R. Thomas. J. Diff. Geom. 54 (2000), no. 2, 367-438.

[42] R. Wendt. J. Geom. Phys. 40, 65-99 (2001)

[43] E. Witten. Nuclear Phys. B 202 (1982), no. 2, 253-316.

[44] E. Witten, J. Differential Geom. 17 (1982), no. 4, 661-692 (1983)

[45] E. Witten, Commun. Math. Phys. 117 (1988) 353-386

[46] E. Witten. Commun. Math. Phys. 121, 351-399 (1989)

[47] E. Witten. J. Geom. Phys. 9, 303-368 (1992)

[48] S. Wu. J. Geom. Phys. 17, 299-309 (1995)

Department of Mathematics, University of California, Santa Cruz, CA 95064

E-mail address: weitsman@math.ucsc.edu 